\title{
Geometric picture of generalized-CP and Higgs-family transformations in the two-Higgs-doublet model
}

\author{
P.M. Ferreira* \\ Instituto Superior de Engenharia de Lisboa, Rua Conselheiro Emídio Navarro, 1900 Lisboa, Portugal and \\ Centro de Física Teórica e Computacional, Faculdade de Ciências, \\ Universidade de Lisboa, Av. Prof. Gama Pinto 2, 1649-003 Lisboa, Portugal \\ Howard E. Haber $\dagger$ \\ Santa Cruz Institute for Particle Physics, University of California, Santa Cruz, California 95064, USA \\ M. Maniatis $\ddagger$ and O. Nachtmann \\ Institut für Theoretische Physik, Philosophenweg 16, 69120 Heidelberg, Germany \\ João P. Silvã \\ Instituto Superior de Engenharia de Lisboa, Rua Conselheiro Emídio Navarro, 1900 Lisboa, Portugal and \\ Centro de Física Teórica de Partículas, Instituto Superior Técnico, P-1049-001 Lisboa, Portugal
}

(Dated: October 30, 2018)

\begin{abstract}
In the two-Higgs-doublet model (THDM), generalized-CP transformations $\left(\varphi_{i} \rightarrow X_{i j} \varphi_{j}^{*}\right.$ where $X$ is unitary) and unitary Higgs-family transformations $\left(\varphi_{i} \rightarrow U_{i j} \varphi_{j}\right)$ have recently been examined in a series of papers. In terms of gauge-invariant bilinear functions of the Higgs fields $\varphi_{i}$, the Higgs-family transformations and the generalized-CP transformations possess a simple geometric description. Namely, these transformations correspond in the space of scalar-field bilinears to proper and improper rotations, respectively. In this formalism, recent results relating generalized CP transformations with Higgs-family transformations have a clear geometric interpretation.
\end{abstract}

\section{INTRODUCTION}

The Standard Model (SM) of particle physics provides an extremely successful framework for describing the prop'erties of the fundamental particles and their interactions. No statistically significant deviation from SM predictions 'has yet been observed in collider experiments 1]. Nevertheless, the experimental exploration of the scalar sector of particle physics is still in its infancy. The SM contains one complex doublet, hypercharge-one multiplet of scalar Higgs 'fields. But it is by no means excluded that the Higgs sector is larger than that of the SM 2]. In particular, there 'are a number of theoretical arguments suggesting a richer Higgs sector than that of the SM. For instance, the twoHiggs-doublet model (THDM) is attractive since it provides a viable framework for spontaneous CP violation [3, 4]. The Peccei-Quinn symmetry, originally introduced in order to solve the so-called strong CP problem [5, 6], requires an enlarged Higgs sector and can be accommodated in the THDM. Typically, supersymmetric models require at least two Higgs-doublet fields [7-11] in order to cancel potential gauge anomalies due to higgsino superpartners. Thus, there is ample motivation to study the simplest two-Higgs-doublet extension of the SM scalar sector.

Indeed, the theory and phenomenology of the THDM has been extensively analyzed; see [2, 12 $[49]$ and references therein. Among these studies of the THDM, one can find two lines of approaches. The traditional approach works

\footnotetext{
*E-mail: ferreira@cii.fc.ul.pt

${ }^{\dagger}$ E-mail: haber@scipp.ucsc.edu

${ }_{\ddagger}^{\ddagger}$ E-mail: m.maniatis@thphys.uni-heidelberg.de

§E-mail: o.nachtmann@thphys.uni-heidelberg.de

IE-mail: jpsilva@cftp.ist.utl.pt
} 
directly with the Higgs-doublet fields 2, 12,36 . In contrast, there is a second approach that emphasizes the role of gauge-invariant scalar field bilinears [37-49]. A systematic use of the scalar field bilinears for the study of the stability and the structure of electroweak symmetry breaking (EWSB) in the THDM was first carried out in [38, 40]. Then independently in [42] the scalar field bilinears were introduced and employed for the study of the CP properties of the THDM. This latter approach was revisited in [43]. In a recent paper [50], three of the present authors mainly employed the traditional approach in the study of Higgs-family and generalized-CP (GCP) symmetries of the THDM. It is the purpose of this work to present a compelling geometrical interpretation of Higgs-family and GCP symmetries using the formalism of scalar field bilinears. This formalism provides a powerful geometric framework that yields new insights and clarifies the relations among the different possible symmetry transformations of the THDM.

In Section 2, we review the formalism of scalar field bilinears and their applications in the analysis of the THDM. In Section 3, we introduce the generalized-CP (GCP) transformations. These transformations are initially defined as transformations of scalar fields. We then revisit the GCP transformations in the formalism of scalar field bilinears and provide a geometric interpretation. Two useful theorems involving GCP transformations are proven in Section 4. Higgs-family and GCP symmetries are examined in detail in Section 5. The classification of all possible THDM symmetries is established in the formalism of scalar field bilinears. The constraints on the scalar potential parameters due to the various possible symmetry classes is provided in a form that is covariant with respect to arbitrary transformations of the basis for the scalar fields. A distinction between parameter constraints derived in an arbitrary basis and in a specific basis is examined and clarified. Conclusions are give in Section 6. Details on the structure of $3 \times 3$ rotation matrices that are useful for the computations performed in this paper are provided in Appendix A.

\section{SCALAR FIELD BILINEARS IN THE THDM}

The scalar sector of the THDM contains two complex doublet, hypercharge-one Higgs fields, with respect to the $\mathrm{SU}(2) \times \mathrm{U}(1)$ electroweak gauge group, denoted by

$$
\varphi_{i}(x)=\left(\begin{array}{c}
\varphi_{i}^{+}(x) \\
\varphi_{i}^{0}(x)
\end{array}\right), \quad \text { with } i=1,2 .
$$

The tree-level THDM Lagrangian contains the kinetic term and the potential $V\left(\varphi_{1}, \varphi_{2}\right)$ for the Higgs fields, which is gauge invariant and renormalizable. These requirements imply that the scalar potential $V\left(\varphi_{1}, \varphi_{2}\right)$ consists only of quadratic and quartic terms in the fields. The conventional parametrization in the field approach reads [2, 18]

$$
\begin{aligned}
V= & m_{11}^{2}\left(\varphi_{1}^{\dagger} \varphi_{1}\right)+m_{22}^{2}\left(\varphi_{2}^{\dagger} \varphi_{2}\right)-m_{12}^{2}\left(\varphi_{1}^{\dagger} \varphi_{2}\right)-\left(m_{12}^{2}\right)^{*}\left(\varphi_{2}^{\dagger} \varphi_{1}\right) \\
& +\frac{1}{2} \lambda_{1}\left(\varphi_{1}^{\dagger} \varphi_{1}\right)^{2}+\frac{1}{2} \lambda_{2}\left(\varphi_{2}^{\dagger} \varphi_{2}\right)^{2}+\lambda_{3}\left(\varphi_{1}^{\dagger} \varphi_{1}\right)\left(\varphi_{2}^{\dagger} \varphi_{2}\right) \\
& +\lambda_{4}\left(\varphi_{1}^{\dagger} \varphi_{2}\right)\left(\varphi_{2}^{\dagger} \varphi_{1}\right)+\frac{1}{2}\left[\lambda_{5}\left(\varphi_{1}^{\dagger} \varphi_{2}\right)^{2}+\lambda_{5}^{*}\left(\varphi_{2}^{\dagger} \varphi_{1}\right)^{2}\right] \\
& +\left[\lambda_{6}\left(\varphi_{1}^{\dagger} \varphi_{2}\right)+\lambda_{6}^{*}\left(\varphi_{2}^{\dagger} \varphi_{1}\right)\right]\left(\varphi_{1}^{\dagger} \varphi_{1}\right)+\left[\lambda_{7}\left(\varphi_{1}^{\dagger} \varphi_{2}\right)+\lambda_{7}^{*}\left(\varphi_{2}^{\dagger} \varphi_{1}\right)\right]\left(\varphi_{2}^{\dagger} \varphi_{2}\right),
\end{aligned}
$$

with $m_{11}^{2}, m_{22}^{2}, \lambda_{1,2,3,4}$ real and $m_{12}^{2}, \lambda_{5,6,7}$ potentially complex. The parameters of the scalar potential must be chosen such that the potential is bounded from below, is stable, and leads to the correct form for EWSB [which preserves $\left.\mathrm{U}(1)_{\mathrm{EM}}\right]$. In addition, one may choose to impose various additional symmetry requirements on the scalar potential.

A very convenient way to study the stability, the structure of the EWSB, and any additional symmetry requirements is to use scalar field bilinears $38-40,42,44]$. We follow here the notation of [38, 40] and define the four independent gauge invariant bilinears as

$$
\begin{array}{ll}
K_{0}=\varphi_{1}^{\dagger} \varphi_{1}+\varphi_{2}^{\dagger} \varphi_{2}, & K_{1}=\varphi_{1}^{\dagger} \varphi_{2}+\varphi_{2}^{\dagger} \varphi_{1}, \\
K_{2}=i \varphi_{2}^{\dagger} \varphi_{1}-i \varphi_{1}^{\dagger} \varphi_{2}, & K_{3}=\varphi_{1}^{\dagger} \varphi_{1}-\varphi_{2}^{\dagger} \varphi_{2} .
\end{array}
$$

We summarize some results from [38, 40]. 
- We have

$$
K_{0} \geq 0, \quad K_{0}^{2}-K_{1}^{2}-K_{2}^{2}-K_{3}^{2} \geq 0 .
$$

That is, the four vectors $\tilde{\mathbf{K}}=\left(K_{0}, \mathbf{K}\right)^{\mathrm{T}}=\left(K_{0}, K_{1}, K_{2}, K_{3}\right)^{\mathrm{T}}$ span the forward light cone in $K$ space. These $\tilde{\mathbf{K}}$ parameterize the gauge orbits of the Higgs-doublet fields.

- A change of basis of the Higgs fields, called a Higgs-family transformation

$$
\left(\begin{array}{l}
\varphi_{1} \\
\varphi_{2}
\end{array}\right) \rightarrow\left(\begin{array}{l}
\varphi_{1}^{\prime} \\
\varphi_{2}^{\prime}
\end{array}\right)=U\left(\begin{array}{l}
\varphi_{1} \\
\varphi_{2}
\end{array}\right),
$$

with $U=\left(U_{i j}\right) \in \mathrm{U}(2)$, corresponds to an $\mathrm{SO}(3)$ rotation in $K$ space

$$
\begin{aligned}
& K_{0} \rightarrow K_{0}^{\prime}=K_{0}, \\
& \mathbf{K} \rightarrow \mathbf{K}^{\prime}=R(U) \mathbf{K} .
\end{aligned}
$$

Here, the $3 \times 3$ real orthogonal matrix $R(U)$ is obtained from

$$
U^{\dagger} \sigma^{a} U=R_{a b}(U) \sigma^{b} .
$$

Since $U$ is continuously connected to the identity, it follows that $\operatorname{det} R(U)=1$. It is straightforward to prove that

$$
R_{a b}(U) R_{b c}(V)=R_{a c}(U V)
$$

starting from eq. (2.7) and using the fact that the $\sigma^{a}$ span the space of traceless $2 \times 2$ Hermitian matrices. Thus, the mapping $\{U,-U\} \longmapsto R(U)$ provides the well-known double cover of $\mathrm{SO}(3)$ by $\mathrm{SU}(2)$. An explicit formula for $R_{a b}(U)$ is easily obtained:

$$
R_{a b}(U)=\frac{1}{2} \operatorname{Tr}\left(U^{\dagger} \sigma^{a} U \sigma^{b}\right) .
$$

The most general $\mathrm{SO}(3)$ matrix can be uniquely specified by an axis of rotation $\hat{\boldsymbol{n}}$ and an angle of rotation $\theta$ that lies in the interval $0 \leq \theta \leq \pi$. We denote the corresponding $3 \times 3$ matrix by $R(\hat{\boldsymbol{n}}, \theta)$. The properties of $R(\hat{\boldsymbol{n}}, \theta)$ are reviewed in Appendix A.

- Every $\mathrm{SO}(3)$ rotation in $K$ space, given by (2.6) with $R \in \mathrm{SO}(3)$, corresponds to a Higgs-family transformation (2.5) that is unique up to gauge transformations.

- The most general gauge invariant and renormalizable potential $V$ of the THDM can be written as

$$
V=\tilde{\mathbf{K}}^{\mathrm{T}} \tilde{\boldsymbol{\xi}}+\tilde{\mathbf{K}}^{\mathrm{T}} \tilde{E} \tilde{\mathbf{K}} .
$$

Here, $\tilde{\boldsymbol{\xi}}$ and $\tilde{E}$ contain the parameters which are all real,

$$
\tilde{\boldsymbol{\xi}}=\left(\begin{array}{c}
\xi_{0} \\
\boldsymbol{\xi}
\end{array}\right), \quad \tilde{E}=\left(\begin{array}{cc}
\eta_{00} & \boldsymbol{\eta}^{T} \\
\boldsymbol{\eta} & E
\end{array}\right),
$$

with $E=E^{\mathrm{T}}$ a $3 \times 3$ matrix. Expressing $\tilde{\boldsymbol{\xi}}$ and $\tilde{\mathbf{E}}$ in terms of the parameters of (2.2), we find

$$
\begin{aligned}
& \xi_{0}=\frac{1}{2}\left(m_{11}^{2}+m_{22}^{2}\right), \quad \boldsymbol{\xi}=\frac{1}{2}\left(\begin{array}{cc}
-2 \operatorname{Re}\left(m_{12}^{2}\right) \\
2 \operatorname{Im}\left(m_{12}^{2}\right) \\
m_{11}^{2}-m_{22}^{2}
\end{array}\right), \quad \boldsymbol{\eta}=\frac{1}{4}\left(\begin{array}{c}
\operatorname{Re}\left(\lambda_{6}+\lambda_{7}\right) \\
-\operatorname{Im}\left(\lambda_{6}+\lambda_{7}\right) \\
\frac{1}{2}\left(\lambda_{1}-\lambda_{2}\right)
\end{array}\right) \\
& \eta_{00}=\frac{1}{8}\left(\lambda_{1}+\lambda_{2}\right)+\frac{1}{4} \lambda_{3}, \quad E=\frac{1}{4}\left(\begin{array}{ccc}
\lambda_{4}+\operatorname{Re}\left(\lambda_{5}\right) & -\operatorname{Im}\left(\lambda_{5}\right) & \operatorname{Re}\left(\lambda_{6}-\lambda_{7}\right) \\
-\operatorname{Im}\left(\lambda_{5}\right) & \lambda_{4}-\operatorname{Re}\left(\lambda_{5}\right) & -\operatorname{Im}\left(\lambda_{6}-\lambda_{7}\right) \\
\operatorname{Re}\left(\lambda_{6}-\lambda_{7}\right) & -\operatorname{Im}\left(\lambda_{6}-\lambda_{7}\right) & \frac{1}{2}\left(\lambda_{1}+\lambda_{2}\right)-\lambda_{3}
\end{array}\right) .
\end{aligned}
$$


- A transformation (2.5) corresponds to a Higgs family symmetry if and only if

$$
R(U) \boldsymbol{\xi}=\boldsymbol{\xi}, \quad R(U) \boldsymbol{\eta}=\boldsymbol{\eta}, \quad R(U) E R^{\mathrm{T}}(U)=E .
$$

In [43] basis changes of the Higgs fields as in eq. (2.5) were considered, but with the unitary transformation $U$ replaced by more general $\mathrm{SL}(2, \mathbb{C})$ transformations. In $K$ space this corresponds to general Lorentz transformations, which includes both rotations and boosts. However, the latter change the form of the kinetic terms of the THDM Lagrangian. Without loss of generality, one may assume that the kinetic terms of the tree-level THDM Lagrangian are of canonical form. Under this assumption, only unitary Higgs family transformations are permitted.

\section{GENERALIZED-CP TRANSFORMATIONS}

In this section we study the generalized-CP (GCP) transformations,

$$
\varphi_{i}(x) \rightarrow X_{i j} \varphi_{j}^{*}\left(x^{\prime}\right), \quad \text { with } i, j \in\{1,2\}, \quad X=\left(X_{i j}\right) \in \mathrm{U}(2), \quad x=\left(\begin{array}{c}
x^{0} \\
\mathbf{x}
\end{array}\right), x^{\prime}=\left(\begin{array}{c}
x^{0} \\
-\mathbf{x}
\end{array}\right) .
$$

Such GCP transformations of scalar fields have been previously considered in [51 55 $]^{1}$, and GCP transformations of fermions fields have also been examined in [58]. A systematic study of GCP transformations of the scalar fields of the THDM was carried out in [45] and [50]. The matrix $X$ that appears in eq. (3.1) is basis-dependent. Under a change of basis specified by eq. (2.5), the GCP transformation of eq. (3.1) is modified to:

$$
\varphi_{i}^{\prime}(x) \rightarrow X_{i j}^{\prime} \varphi_{j}^{\prime *}\left(x^{\prime}\right)
$$

where $X^{\prime}$ is a unitary matrix given by:

$$
X^{\prime} \equiv U X U^{\mathrm{T}}
$$

As shown in [50], three classes of GCP transformations exist depending on the value of (GCP $)^{2}$. Consider first the case of $(\mathrm{GCP})^{2}=1$, which is denoted by $\mathrm{CP} 1$ (sometimes called the "standard" CP symmetry transformation). Then,

$$
\varphi_{i} \rightarrow X_{i j} \varphi_{j}^{*} \rightarrow X_{i j} X_{j k}^{*} \varphi_{k}=\varphi_{i},
$$

which implies that $X X^{*}=\mathbb{1}_{2}$ (where $\mathbb{1}_{2}$ is the $2 \times 2$ identity matrix). Since $X$ is unitary, the latter implies that $X$ is also symmetric. Thus, eq. (3.1) corresponds to a CP1 transformation if and only if $X$ is a symmetric unitary matrix. One can now employ the well known result that any symmetric unitary matrix $X$ can be written as the product of a unitary matrix and its transpose (see e.g. Appendix D.3 of [59] for a proof of this result). That is, one can always find a unitary matrix $U$ such that $X=U^{\dagger} U^{*}$. Performing the basis transformation given by eq. (3.3) then yields that

$$
X^{\prime} \equiv U X U^{\mathrm{T}}=U U^{\dagger}\left(U U^{\dagger}\right)^{*}=\mathbb{1}_{2} .
$$

That is, in the case of $\mathrm{CP} 1$, there is always a basis choice for which $X^{\prime}=\mathbb{1}_{2}$.

Next, consider the case of $(\mathrm{GCP})^{2}=-1$, which is denoted by CP2. Then,

$$
\varphi_{i} \rightarrow X_{i j} \varphi_{j}^{*} \rightarrow X_{i j} X_{j k}^{*} \varphi_{k}=-\varphi_{i}
$$

\footnotetext{
${ }^{1}$ For the discussion of CP transformations in the SM see for example [55 -57].
} 
which implies that $X X^{*}=-\mathbb{1}_{2}$. Since $X$ is unitary, the latter implies that $X$ is also antisymmetric. Thus, eq. (3.1) corresponds to a CP2 transformation if and only if $X$ is an antisymmetric unitary matrix. The most general antisymmetric unitary $2 \times 2$ matrix $X$ is $^{2}$

$$
X=e^{i \psi} \epsilon, \quad \text { where } \epsilon \equiv i \sigma^{2}=-\epsilon^{\mathrm{T}}=-\epsilon^{-1}=\left(\begin{array}{rr}
0 & 1 \\
-1 & 0
\end{array}\right),
$$

where $\psi$ is an arbitrary phase. It then follows that a unitary matrix exists, $U \equiv e^{-i \psi / 2} \epsilon$, such that

$$
X^{\prime}=U X U^{\mathrm{T}}=\epsilon .
$$

That is, in the case of $\mathrm{CP} 2$, there is always a basis choice for which $X^{\prime}=\epsilon$.

Finally, we consider the case of $(\mathrm{GCP})^{2}=X X^{*} \neq \pm \mathbb{1}_{2}$, which is denoted by CP3. In this case, it is always possible to perform a basis transformation such that in the new basis, $X$ is transformed into: ${ }^{3}$

$$
X^{\prime}=U X U^{\mathrm{T}}=\left(\begin{array}{rr}
\cos \theta & \sin \theta \\
-\sin \theta & \cos \theta
\end{array}\right)
$$

where $0<\theta<\pi / 2$.

To prove this result, we first note that since $X$ is a unitary matrix, $\operatorname{det} X \equiv e^{2 i \chi}$ is a pure phase. Following eq. (3.3), we shall perform a basis transformation such that

$$
\operatorname{det} X^{\prime}=\operatorname{det}\left(U X U^{\mathrm{T}}\right)=1 .
$$

This can always be done provided that

$$
U=e^{-i \chi / 2} \widehat{U},
$$

where $\widehat{U}$ is an $\mathrm{SU}(2)$ matrix. It is convenient to define:

$$
\widehat{X} \equiv e^{-i \chi} X
$$

in which case $\operatorname{det} \widehat{X}=1$ and

$$
X^{\prime}=\widehat{U} \widehat{X} \widehat{U}^{\mathrm{T}} .
$$

A general $\mathrm{SU}(2)$ matrix $\widehat{U}$ satisfies:

$$
\widehat{U}=\epsilon \widehat{U}^{*} \epsilon^{-1},
$$

where $\epsilon$ is defined in eq. (3.7). Eq. (3.14) expresses the well known equivalence of the irreducible two-dimensional representation of SU(2) and its complex conjugate. Inserting the transpose of eq. (3.14) into eq. (3.13) yields:

$$
X^{\prime} \epsilon=\widehat{U} \widehat{X} \epsilon \widehat{U}^{\dagger} .
$$

It is convenient to define:

$$
\widehat{U} \equiv \frac{1}{\sqrt{2}}\left(\begin{array}{rr}
1 & 1 \\
i & -i
\end{array}\right) V
$$

\footnotetext{
${ }^{2}$ Conversely, it is straightforward to show that if $X^{\dagger} \sigma^{a} X=-\sigma^{a *}$, where $X=\exp (i \theta \hat{\boldsymbol{n}} \cdot \overrightarrow{\boldsymbol{\sigma}} / 2)$, then $X$ is proportional to $\sigma^{2}$, i.e. $X$ is an antisymmetric unitary matrix.

${ }^{3}$ Eq. [3.9) is an example of a canonical form for unitary congruence. For a comprehensive mathematical treatment, see [60] (note in particular Corollary 8.7). In the physics literature, eq. (3.9) first appeared in [53] and was further generalized in [61].
} 
where $V$ is a unitary matrix (such that $\operatorname{det} V=i$ ). Since $\widehat{X} \epsilon$ is an $\mathrm{SU}(2)$ matrix, it follows that the two eigenvalues of $\widehat{X} \epsilon$ are complex conjugates of each other, denoted below by $e^{ \pm i \phi}$, where the real angle $\phi$ is defined modulo $\pi$. Then, we can choose $V$ to be the unitary matrix that diagonalizes $\widehat{X} \epsilon$,

$$
V \widehat{X} \epsilon V^{\dagger}=\left(\begin{array}{cc}
e^{i \phi} & 0 \\
0 & e^{-i \phi}
\end{array}\right)
$$

Inserting eqs. (3.16) and (3.17) into eq. (3.15) yields

$$
X^{\prime}=\left(\begin{array}{rr}
\sin \phi & -\cos \phi \\
\cos \phi & \sin \phi
\end{array}\right) .
$$

Finally, we define $\phi=\theta+\pi / 2$ to obtain the desired form given by eq. (3.9).

The angle $\phi$ (and hence the angle $\theta$ ) is defined modulo $\pi$. Thus, it is convenient to establish a convention in which $|\theta| \leq \pi / 2$. However, we are free to redefine $U \rightarrow \sigma^{1} U$, which has the effect of changing the overall sign of $\theta .^{4}$ Consequently, it is always possible to find a basis transformation such that $X^{\prime}$ takes the form given by eq. (3.9), where $0 \leq \theta \leq \pi / 2$. Moreover,

$$
X X^{*}=U^{\dagger} X^{\prime} U^{*} U^{\mathrm{T}} X^{\prime *} U=U^{\dagger}\left(\begin{array}{rr}
\cos 2 \theta & \sin 2 \theta \\
-\sin 2 \theta & \cos 2 \theta
\end{array}\right) U
$$

so that $\theta=0$ corresponds to the case of CP1 [eq. (3.5)], $\theta=\pi / 2$ corresponds to the case of CP2 [eq. (3.8)], and $0<\theta<\pi / 2$ corresponds to the case of CP3 [eq. (3.9)] .

Summarizing the above results, it follows that in a suitable basis for the scalar fields the matrix $X$ in (3.1) can always be brought to the form

$$
\left(\begin{array}{rr}
\cos \theta & \sin \theta \\
-\sin \theta & \cos \theta
\end{array}\right), \quad \text { with } 0 \leq \theta \leq \pi / 2 .
$$

The classification of GCP symmetries established above is [50]:

- $\mathrm{CP} 1$ if $\theta=0$,

- CP2 if $\theta=\pi / 2$ and

- CP3 if $0<\theta<\pi / 2$.

We now demonstrate how the classification of GCP symmetries can be understood in the formalism of field bilinears employed in [45]. To make the present paper self-contained, we shall repeat some of the derivations of [45] in the analysis that follows.

In the notation of eq. (2.7), we define the $\mathrm{SO}(3)$ matrix $R_{a b}(X)$ via:

$$
X^{\dagger} \sigma^{a} X=R_{a b}(X) \sigma^{b}
$$

where $X$ is the unitary matrix that specifies the GCP transformation [cf. eq. [3.1)]. It is convenient to introduce the improper rotation matrix,

$$
\bar{R}_{2} \equiv \operatorname{diag}(1,-1,1)
$$

\footnotetext{
${ }^{4}$ For any $2 \times 2$ matrix $A$, the matrix $\sigma^{1} A \sigma^{1}$ is related to $A$ by an interchange of the two diagonal elements and an interchange of the two off-diagonal elements.
} 
The matrix $\bar{R}_{2}$ describes the reflection through the 1-3 plane in $K$ space. Similarly, we introduce $\bar{R}_{1}$ and $\bar{R}_{3}$ as the reflections through the $2-3$ and $1-2$ planes, respectively, ${ }^{5}$

$$
\begin{aligned}
& \bar{R}_{1}=\operatorname{diag}(-1,1,1), \\
& \bar{R}_{3}=\operatorname{diag}(1,1,-1) .
\end{aligned}
$$

In particular, note that

$$
\sigma^{a *}=\sigma^{a T}=\left(\bar{R}_{2}\right)_{a b} \sigma^{b} .
$$

The scalar field bilinears of eq. (2.3) can be rewritten as:

$$
K_{\mu}=\varphi_{i}^{\dagger} \sigma_{i j}^{\mu} \varphi_{j}
$$

where $\sigma^{\mu}=(1, \overrightarrow{\boldsymbol{\sigma}})$. Then, the GCP transformation defined in eq. (3.1) corresponds to

$$
K_{\mu}=\varphi_{i}^{\dagger} \sigma_{i j}^{\mu} \varphi_{j} \rightarrow\left(X_{i k} \varphi_{k}^{\dagger}\right)^{*} \sigma_{i j}^{\mu} X_{j \ell} \varphi_{\ell}^{*}=\varphi_{\ell}^{\dagger} \varphi_{k}\left(X^{\dagger} \sigma^{\mu} X\right)_{k \ell} .
$$

If $\mu=0$, then $X^{\dagger} \sigma^{0} X=X^{\dagger} X=\mathbb{1}_{2}$, and so $K_{0} \rightarrow K_{0}$ (where we suppress the coordinates $x$ and $x^{\prime}$ ). If $\mu=a=1,2,3$, then one may use eqs. (3.21) and (3.24) to obtain:

$$
\begin{aligned}
K_{a} & \rightarrow \varphi_{\ell}^{\dagger} \varphi_{k}\left(X^{\dagger} \sigma^{a} X\right)_{k \ell} \\
& =\varphi_{\ell}^{\dagger} \varphi_{k} R_{a b}(X) \sigma_{k \ell}^{b} \\
& =\varphi_{\ell}^{\dagger} \varphi_{k} R_{a b}(X)\left(\bar{R}_{2}\right)_{b c} \sigma_{\ell k}^{c} \\
& =\bar{R}_{a c} K_{c},
\end{aligned}
$$

where $\bar{R}$ is the improper rotation matrix:

$$
\bar{R} \equiv R(X) \bar{R}_{2}
$$

That is,

$$
\begin{aligned}
K_{0}(x) & \rightarrow K_{0}\left(x^{\prime}\right), \\
\mathbf{K}(x) & \rightarrow \bar{R} \mathbf{K}\left(x^{\prime}\right),
\end{aligned}
$$

which reproduces the result obtained in section 3 of [45].

Under each of the three classes of GCP transformations, the improper rotation matrix $\bar{R} \equiv R(X) \bar{R}_{2}$ must satisfy an appropriate constraint equation. To derive the relevant constraint, we start with the complex conjugate of eq. (3.21). Using eq. (3.24), it then follows that

$$
X^{\mathrm{T}}\left(\bar{R}_{2}\right)_{a b} \sigma^{b} X^{*}=R_{a b}(X)\left(\bar{R}_{2}\right)_{b c} \sigma^{c}
$$

Employing eq. (3.21) once more yields

$$
\left(\bar{R}_{2}\right)_{a b} R_{b c}\left(X^{*}\right) \sigma^{c}=R_{a b}(X)\left(\bar{R}_{2}\right)_{b c} \sigma^{c} .
$$

Since the $\sigma^{a}$ are linearly independent and span the space of traceless $2 \times 2$ Hermitian matrices, eq. (3.31) yields:

$$
R\left(X^{*}\right)=\bar{R}_{2} R(X) \bar{R}_{2}
$$

\footnotetext{
${ }^{5}$ Here and in the following $R, R_{\alpha}, \ldots$ and $\bar{R}, \bar{R}_{\alpha}, \ldots$ denote proper and improper rotation matrices with determinant +1 and -1 , respectively.
} 
after using $\left(\bar{R}_{2}\right)^{2}=\mathbb{1}_{3}$ (where $\mathbb{1}_{3}$ is the $3 \times 3$ identity matrix). Finally, we multiply eq. (3.32) on the left by $R(X)$ and make use of eq. (2.8) to obtain:

$$
R\left(X X^{*}\right)=\bar{R}^{2}
$$

where the improper rotation matrix $\bar{R} \equiv R(X) \bar{R}_{2}$ was introduced in eq. (3.28).

Consider separately the cases CP2 (where $X$ is antisymmetric and $X X^{*}=-\mathbb{1}_{2}$ ) and CP1 (where $X$ is symmetric and $X X^{*}=\mathbb{1}_{2}$ ). In both cases, eq. (3.21) yields $R\left(X X^{*}\right)=R\left( \pm \mathbb{1}_{2}\right)=\mathbb{1}_{3}$, and it follows that $\bar{R}^{2}=\mathbb{1}_{3}$. That is, $\bar{R}$ is either a reflection matrix corresponding to a reflection through some plane in $K$ space or an inversion (or point reflection) through the origin in $K$ space. In eq. (3.7), we noted the most general form for $X$ in the case of CP2 is given by $X=e^{i \psi} i \sigma^{2}$, where $\psi$ is an arbitrary phase. Inserting this result into eq. (3.21) and making use of eq. (3.24) yields:

$$
R_{a b}(X) \sigma^{b}=\sigma^{2} \sigma^{a} \sigma^{2}=-\sigma^{a *}=-\left(\bar{R}_{2}\right)_{a b} \sigma^{b},
$$

and we conclude that $R(X)=-\bar{R}_{2}$. Hence, eq. (3.28) yields $\bar{R}=-\mathbb{1}_{3}$, which corresponds to an inversion (i.e., a point reflection through the origin in $K$ space). This case is a $\mathrm{CP}_{g}^{(i)}$ transformation in the notation of [45]. In contrast, for the case of CP1, where $X$ is a symmetric unitary matrix, we have $\bar{R}^{2}=\mathbb{1}_{3}$ and $\bar{R} \neq-\mathbb{1}_{3}$. An improper rotation matrix of this type corresponds to reflections through planes in $K$ space. In particular, an $\mathrm{SO}(3)$ matrix $\widetilde{R}$ exists such that

$$
\bar{R}=\widetilde{R} \bar{R}_{2} \widetilde{R}^{\mathrm{T}}
$$

To prove eq. (3.35), simply choose a basis where $X=\mathbb{1}_{2}$ in which case $R(X)=\mathbb{1}_{3}$ and $\bar{R}=\bar{R}_{2}$. Then, rotate in $K$ space to an arbitrary basis using the rotation matrix $\widetilde{R}$ to obtain eq. (3.35). One can easily check that $\bar{R}^{2}=\mathbb{1}_{3}$ and $\bar{R} \neq-\mathbb{1}_{3}$ as required. This case corresponds to a $\mathrm{CP}_{g}^{(i i)}$ transformation in the notation of [45]. In summary, for those GCP transformations whose square is equal to the unit transformation when acting on the gauge invariant field bilinears, we must have $\bar{R}^{2}=\mathbb{1}_{3}$. The resulting classification of [45] is then related to that of [50] as follows:

- $\mathrm{CP}_{g}^{(i i)}$ : reflections through planes in $K$ space, $\bar{R}=\tilde{R} \bar{R}_{2} \tilde{R}^{\mathrm{T}}$ with $\tilde{R} \in \mathrm{SO}(3) \quad \Longleftrightarrow \quad \mathrm{CP} 1$ transformations.

- $\mathrm{CP}_{g}^{(i)}$ : a point reflection through the origin in $K$ space, $\bar{R}=-\mathbb{1}_{3} \quad \Longleftrightarrow \quad \mathrm{CP} 2$ transformations.

The case of CP3 transformations was not considered in detail in [45]. In this case, $X X^{*} \neq \pm \mathbb{1}_{2}$, which implies that $R\left(X X^{*}\right) \neq \mathbb{1}_{3}$. Hence, eq. (3.33) yields $\bar{R}^{2} \neq \mathbb{1}_{3}$, which means that the improper rotation $\bar{R}$ is not a reflection matrix or an inversion. To obtain an explicit form for $\bar{R} \equiv R(X) \bar{R}_{2}$, it is convenient to choose a suitable basis in which $X$ is given by eq. (3.20), which can be rewritten as:

$$
X=\mathbb{1}_{2} \cos \theta+i \sigma^{2} \sin \theta .
$$

We can use eq. (2.9) to obtain the corresponding rotation matrix $R_{a b}(X)$. Evaluating the relevant traces, one obtains:

$$
R_{a b}(X)=\delta_{a b} \cos 2 \theta+2 \delta_{a 2} \delta_{b 2} \sin ^{2} \theta+\epsilon_{a b 2} \sin 2 \theta .
$$

Hence, in a basis in which $X$ is given by eq. (3.20),

$$
\bar{R} \equiv R(X) \bar{R}_{2}=\left(\begin{array}{crc}
\cos 2 \theta & 0 & -\sin 2 \theta \\
0 & -1 & 0 \\
\sin 2 \theta & 0 & \cos 2 \theta
\end{array}\right) .
$$

As expected, for a CP1 transformation $\theta=0$ and $\bar{R}=\bar{R}_{2}$ [in a suitable basis in which $X=\mathbb{1}_{2}$ ], and for a CP2 transformation $\theta=\pi / 2$ and $\bar{R}=-\mathbb{1}_{3}$. The case of $0<\theta<\pi / 2$ corresponds to a CP3 transformation, in which $\bar{R}$ is an improper rotation matrix that is not a simple reflection or inversion (i.e. $\bar{R}^{2} \neq \mathbb{1}_{3}$ ). As in eq. (3.35), the form for $\bar{R}$ in a general basis is related to eq. (3.38) by an orthogonal similarity transformation,

$$
\bar{R}=\widetilde{R} R(X) \bar{R}_{2} \widetilde{R}^{\mathrm{T}}
$$


for some $\mathrm{SO}(3)$ matrix $\widetilde{R}$, where $R(X)$ is given by eq. (3.37).

Thus, we have reproduced above the result proved in [45]. Every transformation of the field bilinears given by eq. (3.29), where $\bar{R}$ is any improper rotation matrix, corresponds to a GCP transformation of the fields as specified in eq. (3.1). This field transformation is uniquely determined by $\bar{R}$ up to gauge transformations. In analogy with eq. (2.13), a GCP transformation of the form given by eq. (3.29) corresponds to a GCP symmetry if and only if

$$
\bar{R} \boldsymbol{\xi}=\boldsymbol{\xi}, \quad \bar{R} \boldsymbol{\eta}=\boldsymbol{\eta}, \quad \bar{R} E \bar{R}^{\mathrm{T}}=E .
$$

\section{TWO THEOREMS INVOLVING GCP TRANSFORMATIONS}

In [50], it was suggested that all symmetries of the THDM could be expressed in terms of products of GCP symmetries. In this section, we prove two simple theorems that demonstrate that all Higgs family and GCP transformations can be expressed in terms of products of CP1 transformations.

Theorem 1: Any Higgs-family transformation can be considered as a product of two CP1 transformations.

Theorem 2: Any GCP transformation is either a CP1 transformation or a product of three CP1 transformations.

These theorems are new. Some particular cases were considered in [50] but even there they referred only to symmetries where the form of the potential could play a role. The results presented here apply to the more fundamental transformations themselves.

Proof of Theorem 1. Let $X$ and $Y$ be two arbitrary symmetric unitary matrices. Consider the product of the corresponding CP1 transformations,

$$
\begin{aligned}
\varphi_{j}^{\prime}(x) & =X_{j k} \varphi_{k}^{*}\left(x^{\prime}\right), \\
\varphi_{i}^{\prime \prime}(x) & =Y_{i j} \varphi_{j}^{\prime *}\left(x^{\prime}\right)=Y_{i j} X_{j k}^{*} \varphi_{k}(x)=U_{i k} \varphi_{k}(x),
\end{aligned}
$$

where $U=Y X^{*}$ is a unitary matrix. The theorem is proven if we can show that an arbitrary unitary matrix $U$ is the product of two symmetric unitary matrices. But this last statement is easy to prove. First, we diagonalize $U$ with a unitary matrix $W$,

$$
U=W D W^{\dagger}
$$

where $D$ is a diagonal matrix of phases corresponding to the eigenvalues of $U$. Then define the following two symmetric unitary matrices:

$$
S_{1} \equiv W W^{\mathrm{T}}, \quad S_{2} \equiv W^{*} D W^{\dagger}
$$

It immediately follows that

$$
S_{1} S_{2}=W W^{\mathrm{T}} W^{*} D W^{\dagger}=W D W^{\dagger}=U
$$

which shows that any unitary matrix can be written as a product of two symmetric unitary matrices.

It is also instructive to prove Theorem 1 in $K$ space. Consider an arbitrary Higgs-family transformation [cf. eqs. (2.5) and [2.6)]. Every proper rotation matrix $R$ is a rotation about an axis and can be represented, in a suitable basis, as

$$
R_{\alpha}=\left(\begin{array}{ccc}
\cos \alpha & -\sin \alpha & 0 \\
\sin \alpha & \cos \alpha & 0 \\
0 & 0 & 1
\end{array}\right), \quad \text { with } 0 \leq \alpha \leq \pi
$$

This rotation can also be generated by two reflections, $\bar{R}_{\alpha / 2}$ and $\bar{R}_{\alpha}$, as illustrated in Fig. 1. 


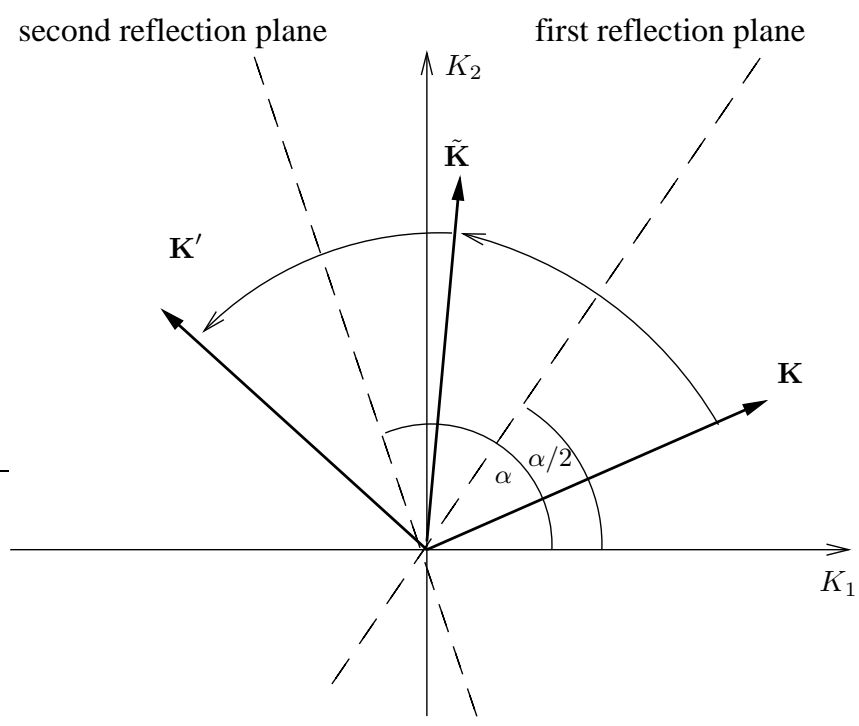

FIG. 1: The reflections $\bar{R}_{\alpha / 2}$ and $\bar{R}_{\alpha}$ illustrated in the $K_{1}-K_{2}$ plane. The reflection $\bar{R}_{\alpha / 2}$ brings the arbitrary vector $\mathbf{K}$ to $\tilde{\mathbf{K}}$. Then, $\bar{R}_{\alpha}$ brings $\tilde{\mathbf{K}}$ to $\mathbf{K}^{\prime}$.

The first reflection, $\bar{R}_{\alpha / 2}$, is through the plane containing the $K_{3}$ axis and the line of the angle $\alpha / 2$ in the $K_{1}-K_{2}$ plane. The second reflection, $\bar{R}_{\alpha}$, is through the plane containing the $K_{3}$ axis and the line of the angle $\alpha$ itself. The arbitrary vector $\mathbf{K}$ goes into $\widetilde{\mathbf{K}}$ under the reflection $\bar{R}_{\alpha / 2}$ and further into $\mathbf{K}^{\prime}$ under $\bar{R}_{\alpha}$. In Fig. 1 we show the projections of these vectors onto the $K_{1}-K_{2}$ plane. Since the third component of the vectors are not changed under $\bar{R}_{\alpha / 2}$ and $\bar{R}_{\alpha}$ we see that $\mathbf{K}^{\prime}$ gives exactly the vector rotated by the angle $\alpha$, that is, $\mathbf{K}^{\prime}=R_{\alpha} \mathbf{K}$, where $R_{\alpha}$ is given by eq. (4.6). To see all this formally we consider $R_{\beta}$ as in eq. (4.6) but with $\alpha$ replaced by an arbitrary angle $\beta$ and define

$$
\bar{R}_{\beta}=R_{\beta} \bar{R}_{2} R_{\beta}^{\mathrm{T}}=\left(\begin{array}{ccc}
\cos 2 \beta & \sin 2 \beta & 0 \\
\sin 2 \beta & -\cos 2 \beta & 0 \\
0 & 0 & 1
\end{array}\right) .
$$

Note that $\left(\bar{R}_{\beta}\right)^{2}=\mathbb{1}_{3}$, which indicates that $\bar{R}_{\beta}$ is a pure reflection. In particular, $\bar{R}_{\beta}$ describes the reflection through the plane containing the $K_{3}$ axis and the line of angle $\beta$ in the $K_{1}-K_{2}$ plane. We have $R_{\beta}^{\mathrm{T}}=R_{-\beta}, \bar{R}_{2} R_{\beta} \bar{R}_{2}=R_{-\beta}$, and $R_{\beta_{1}} R_{\beta_{2}}=R_{\beta_{1}+\beta_{2}}$. It is then easy to see that we get $R_{\alpha}$ from eq. (4.6) via two reflections,

$$
\bar{R}_{\alpha} \bar{R}_{\alpha / 2}=R_{\alpha} \bar{R}_{2} R_{\alpha}^{\mathrm{T}} R_{\alpha / 2} \bar{R}_{2} R_{\alpha / 2}^{\mathrm{T}}=R_{\alpha} .
$$

This is the $K$ space equivalence of the statement that any Higgs family transformation is equivalent to the product of two CP1 transformations.

Proof of Theorem 2. If we perform three successive CP1 transformations of the form given by eq. (3.1), with $X$ given by $X_{1}, X_{2}$ and $X_{3}$, respectively, then the resulting transformation is of the form of eq. (3.1) with

$$
X=X_{3} X_{2}^{*} X_{1},
$$

where the $X_{i}$ are symmetric unitary matrices. However, any unitary matrix $X$ can be written in the form of eq. (4.9). This follows from the fact that any unitary matrix can be written as the product of two symmetric unitary matrices, as proven in eq. (4.5). Thus, one can simply choose one of the matrices $X_{i}$ in eq. (4.9) to be the identity matrix ${ }^{6}$ (which

\footnotetext{
${ }^{6}$ Of course, this does not imply that a GCP transformation can be expressed as the product of two CP1 transformations. Each CP1 transformation involves the complex conjugation of the scalar fields, so one requires a product of odd number of CP1 transformations to express a GCP transformation. The product of two CP1 transformations is a Higgs family transformation as demonstrated in Theorem 1.
} 
is of course a symmetric unitary matrix). Finally, we note that the product of an odd number of GCP transformations is a GCP transformation, as this follows trivially from eq. (3.1). The proof of Theorem 2 is then complete.

Again, it is instructive to prove Theorem 2 in $K$ space. Consider an arbitrary GCP transformation specified by eq. (3.29). In a suitable basis $\bar{R}$ has the form in eq. (3.38). For $\theta=0$ we are finished, since $\bar{R}=\bar{R}_{2}$ which corresponds to a $\mathrm{CP} 1$ transformation. For $\theta \neq 0$ we make a basis transformation in eq. (3.38) exchanging the 2 and the 3 axes, which yields an improper rotation,

$$
\bar{R}_{2 \theta}^{\prime}=\left(\begin{array}{ccr}
\cos 2 \theta & -\sin 2 \theta & 0 \\
\sin 2 \theta & \cos 2 \theta & 0 \\
0 & 0 & -1
\end{array}\right)
$$

We can represent this matrix as a product of three CP1 transformations by multiplying eq. (4.8) by $\bar{R}_{3}$ and replacing $\alpha$ by $2 \theta$. Thus we obtain

$$
\bar{R}_{3} \bar{R}_{2 \theta} \bar{R}_{\theta}=\left(\widetilde{R} \bar{R}_{2} \widetilde{R}^{\mathrm{T}}\right)\left(R_{2 \theta} \bar{R}_{2} R_{2 \theta}^{\mathrm{T}}\right)\left(R_{\theta} \bar{R}_{2} R_{\theta}^{\mathrm{T}}\right)=\bar{R}_{2 \theta}^{\prime}
$$

where

$$
\widetilde{R}=\left(\begin{array}{rrr}
1 & 0 & 0 \\
0 & 0 & -1 \\
0 & 1 & 0
\end{array}\right)
$$

Eq. (4.11) is the $K$ space equivalence of the statement that an arbitrary GCP transformation either is a CP1 transformation or the product of three CP1 transformations.

Note that Theorem 2 is equivalent to the statement that an arbitrary improper $3 \times 3$ rotation matrix can be expressed as a product of three reflection matrices. This statement can be proved directly. First, we note that any proper $3 \times 3$ rotation matrix can be written as the product of two reflection matrices as in eq. (4.8). However, any improper rotation matrix can be written as the product of a reflection matrix and a proper rotation matrix. Combining these two statements yields the desired result. ${ }^{7}$

\section{HIGGS-FAMILY AND GCP SYMMETRIES}

In Sections 3 and 4, we analyzed Higgs-family and GCP transformations. In this section, we shall address the corresponding symmetries. In the scalar sector of the THDM, there are six nontrivial inequivalent classes of symmetries that can be exhibited by the tree-level THDM scalar potential. The complete classification of the THDM symmetry classes, which are listed in Table I, was first given in [44] and subsequently analyzed in [50].

The following points are noteworthy. First, consider the case in which a Higgs-family or a GCP transformation is a symmetry transformation of the THDM. The representation of such a symmetry transformation as a product of other transformations, (e.g. a product of CP1 transformations as discussed in Section 4), does not automatically imply that the individual factors of the product are also symmetries of the THDM. Second, the analysis of this paper is confined to the scalar sector of the THDM. Ultimately, one must also include the Yukawa couplings to fermions in the theory in the discussion of symmetry transformations. The structure of the symmetries of the scalar sector may not be respected by the Yukawa sector. As an example, consider a field transformation $S_{1}$ that can be written as a product of two others, $S_{1}=S_{2} S_{3}$. Suppose that by imposing the symmetry $S_{1}$, the symmetries $S_{2}$ and $S_{3}$ are automatically respected by the scalar sector. This property is not guaranteed to hold for the Yukawa sector. In particular, it is possible that imposing $S_{2}$ and $S_{3}$ separately as symmetries may lead to stronger restrictions as compared with the imposition of the symmetry $S_{1}$ by itself. This is indeed the case in the model which was studied in [46, 47, 49].

\footnotetext{
${ }^{7}$ In particular, the inversion matrix $-\mathbb{1}_{3}$ can be written as the product of three reflection matrices. Starting from eq. 4.8), we note that $-R_{\alpha}^{\mathrm{T}}$ is a reflection matrix, in which case, $-R_{\alpha}^{\mathrm{T}} \bar{R}_{\alpha} \bar{R}_{\alpha / 2}=-\mathbb{1}_{3}$.
} 


\begin{tabular}{lll}
\hline symmetry class & rotation matrices in a generic basis & rotation matrices as a product of CP1 reflection matrices \\
\hline $\mathbb{Z}_{2}$ & $R(\hat{\boldsymbol{n}}, \pi)$ & $R_{\pi}=\bar{R}_{2} \bar{R}_{1}$, for $\hat{\boldsymbol{n}}=\hat{\boldsymbol{z}}$ [see eq. (5.4)] \\
$\mathrm{U}(1)$ & $R(\hat{\boldsymbol{n}}, \theta), \quad(0<\theta<\pi)$ & $R_{2 \theta}=\bar{R}_{2 \theta} \bar{R}_{\theta}$, for $\hat{\boldsymbol{n}}=\hat{\boldsymbol{z}}$ [see eq. (5.23)] \\
$\mathrm{SO}(3)$ & $\pm R\left(\hat{\boldsymbol{n}}_{1}, \theta\right), \pm R\left(\hat{\boldsymbol{n}}_{2}, \theta\right),\left(\hat{\boldsymbol{n}}_{1} \times \hat{\boldsymbol{n}}_{2} \neq 0\right)$ & See section [5.3] \\
$\mathrm{CP} 1$ & $-R(\hat{\boldsymbol{n}}, \pi)$ & $\bar{R}_{2}$, for $\hat{\boldsymbol{n}}=\hat{\boldsymbol{y}}$ [see eq. (5.34)] \\
$\mathrm{CP} 2$ & $-R(\hat{\boldsymbol{n}}, 0)=-\mathbb{1}_{3}$ & $-\mathbb{1}_{3}=\bar{R}_{3} \bar{R}_{2} \bar{R}_{1}$ [see eq. (5.44)] \\
$\mathrm{CP} 3$ & $-R(\hat{\boldsymbol{n}}, \theta), \quad(0<\theta<\pi)$ & $\bar{R}_{2 \theta_{1}}^{\prime}=\bar{R}_{3} \bar{R}_{2 \theta_{1}} \bar{R}_{\theta_{1}}$, for $\hat{\boldsymbol{n}}=\hat{\boldsymbol{z}}$ [see eq. (5.46)] \\
\hline
\end{tabular}

TABLE I: The symmetry classes and the corresponding proper [and improper] rotation matrices $R$ [and $\bar{R}$ ] that generate the symmetry classes in $K$ space. A general rotation matrix $R(\hat{\boldsymbol{n}}, \theta)$ is uniquely determined by an axis of rotation $\hat{\boldsymbol{n}}$ and rotation angle $\theta$ (see Appendix A). The identity class generated by $R(\hat{\boldsymbol{n}}, 0)=\mathbb{1}_{3}$ is trivial and is not explicitly displayed. The decomposition of $R$ and $\bar{R}$ in terms of products of CP1 reflection matrices is also given in a particular basis. In the case of the $\mathrm{CP} 2$ and $\mathrm{SO}(3)$ symmetry classes, the corresponding rotation matrices are invariant with respect to basis transformations. The generation of the $\mathrm{SO}(3)$ symmetry class requires two rotation matrices (each of which may be a proper or improper rotation depending on the overall sign choice), where $\hat{\boldsymbol{n}}_{1}$ and $\hat{\boldsymbol{n}}_{2}$ are non-collinear.

\subsection{Symmetries and single rotations in bilinear space}

We shall now use Theorems 1 and 2 of Section 4 to obtain a new derivation of the statement (given in [50]) that all possible symmetries of the scalar sector of the THDM can be reduced to multiple applications of the standard CP symmetry in suitable bases. In Table \ we list the possible classes of THDM symmetries, along with the equivalent result in $K$ space, where the Higgs family and generalized CP transformations are generated by proper or improper rotations, $R$ or $\bar{R}$, respectively. Also shown is the decomposition of $R$ and $\bar{R}$ in terms of products of CP1 reflection matrices in a particular basis choice. ${ }^{8}$ Note that it is sufficient to require the invariance under a single but suitable rotation in order to generate each symmetry class, with the exception of the $\mathrm{SO}(3)$ case. $^{9}$

The transformation matrices $R$ and $\bar{R}$ generating the respective symmetry classes can be written as products of CP1 transformations using Theorems 1 and 2 of Section 4. Of course, one still needs to check if the imposition of the $\mathrm{CP} 1$ factors as symmetries is equivalent or more restrictive than the corresponding proper or improper rotation, $R$ or $\bar{R}$, alone. Let us now derive and discuss the results shown in Table \in detail.

\section{- $\mathbb{Z}_{2}$ symmetry}

The $\mathbb{Z}_{2}$ symmetry [62, 63],

$$
\begin{aligned}
\varphi_{1}(x) & \rightarrow \varphi_{1}(x), \\
\varphi_{2}(x) & \rightarrow-\varphi_{2}(x),
\end{aligned}
$$

corresponds in $K$ space to a rotation by $\pi$ around the third axis,

$$
\mathbf{K}(x) \rightarrow R_{\pi} \mathbf{K}(x),
$$

where $R_{\pi}$ is as in eq. (4.6) with $\alpha=\pi$. That is,

$$
R_{\mathbb{Z}_{2}}=R_{\pi}=\left(\begin{array}{rrr}
-1 & 0 & 0 \\
0 & -1 & 0 \\
0 & 0 & 1
\end{array}\right) .
$$

\footnotetext{
${ }^{8}$ In the CP2 symmetry class, the rotation matrices are independent of $\hat{\boldsymbol{n}}$, so that the basis choice in this case is fixed by the direction of an eigenvector of $E$ corresponding to one of its non-degenerate eigenvalues.

${ }^{9}$ For the $\mathrm{SO}(3)$ symmetry class, if a basis for the scalar fields is appropriately chosen, then it is possible to generate the $\mathrm{SO}(3)$ symmetry class via a single rotation matrix, as shown in Section 5.3
} 
This result is most easily obtained by taking $U=\sigma^{3}$ in eq. (2.9).

The application of Theorem 1 gives the decomposition [cf. eq. [4.8] ]:

$$
R_{\pi}=\bar{R}_{\pi} \bar{R}_{\pi / 2}=\bar{R}_{2} \bar{R}_{1}
$$

Requiring $\mathbb{Z}_{2}$ to be a symmetry means that the THDM parameters specified in eq. (2.12) must satisfy eq. (2.13) with $R(U)$ replaced by $R_{\pi}$, which yields:

$$
\xi_{1}=\xi_{2}=0, \quad \eta_{1}=\eta_{2}=0, \quad E_{13}=E_{23}=0 .
$$

On the other hand, requiring the $\mathrm{CP} 1$ transformations corresponding to both $\bar{R}_{1}$ and $\bar{R}_{2}$ to be separate symmetries gives, in addition to eq. (5.5),

$$
E_{12}=0 .
$$

Thus, imposing the CP1 symmetries $\bar{R}_{1}$ and $\bar{R}_{2}$ yields a stronger constraint than the $\mathbb{Z}_{2}$ symmetry alone. Nevertheless, the scalar potential subject to the CP1 symmetries $\bar{R}_{1}$ and $\bar{R}_{2}$ is physically equivalent to the scalar potential subject to the $\mathbb{Z}_{2}$ symmetry, since the two scalar potentials are relate by a change of basis. This is easily proved by performing a change of basis characterized by an $\mathrm{SO}(3)$ matrix $R$ [cf. eq. (2.6)] with $R_{13}=R_{23}=R_{31}=R_{32}=0$ and $R_{33}=1$. Note that $\boldsymbol{\xi}$ and $\boldsymbol{\eta}$ are invariant under this change of basis, whereas $E \rightarrow R E R^{\mathrm{T}}$. One can choose $R$ such that $E$ is diagonal, which confirms that the two scalar potentials are related by a basis transformation.

It is also instructive to introduce the permutation symmetry $\Pi_{2}$,

$$
\begin{aligned}
& \varphi_{1}(x) \rightarrow \varphi_{2}(x), \\
& \varphi_{2}(x) \rightarrow \varphi_{1}(x) .
\end{aligned}
$$

In fact, the $\mathbb{Z}_{2}$-symmetric scalar potential and the $\Pi_{2}$-symmetric scalar potential are related by a basis transformation [25, 50] (and are hence physically equivalent). To obtain the $K$ space description of $\Pi_{2}$, simply insert $U=\sigma^{1}$ into eq. (2.9), which yields:

$$
R_{\Pi_{2}}=\left(\begin{array}{rrr}
1 & 0 & 0 \\
0 & -1 & 0 \\
0 & 0 & -1
\end{array}\right) .
$$

Requiring $\Pi_{2}$ to be a symmetry implies that the THDM parameters specified in eq. (2.12) must satisfy eq. (2.13) with $R(U)$ replaced by $R_{\Pi_{2}}$, which yields:

$$
\xi_{2}=\xi_{3}=0, \quad \eta_{2}=\eta_{3}=0, \quad E_{12}=E_{13}=0 .
$$

It is possible (although not particularly illuminating) to construct the basis transformation that relates eqs. (5.5) and (5.9). However, it is more useful to identify the most general transformation in $K$ space that corresponds to the presence of the $\mathbb{Z}_{2}$ symmetry specified by eq. (5.1) in some basis. This can be accomplished by starting in a basis where eq. (5.2) is satisfied and transforming to an arbitrary basis.

Under a basis transformation specified by the $\mathrm{U}(2)$ matrix $U$ [cf. eqs. (2.5) and (2.6)], we define $\widetilde{R} \equiv R(U)$, where $R(U)$ is given by eq. (2.9). Then, the $\mathbb{Z}_{2}$ symmetry transformation, $\mathbf{K}(x) \rightarrow R_{\pi} \mathbf{K}(x)$, is transformed to

$$
\widetilde{R} \mathbf{K}(x) \rightarrow \widetilde{R} R(\hat{\boldsymbol{z}}, \pi) \widetilde{R}^{\mathrm{T}} \widetilde{R} \mathbf{K}(x),
$$

where $R_{\pi} \equiv R(\hat{z}, \pi)$ is a rotation by $180^{\circ}$ about the $z$-axis, and the expression $\widetilde{R}^{\mathrm{T}} \widetilde{R}=\mathbb{1}_{3}$ has been conveniently inserted. Using eq. A.20 given in Appendix A, it follows that

$$
\mathbf{K}^{\prime}(x) \equiv \widetilde{R} \mathbf{K}(x)=R(\hat{\boldsymbol{n}}, \pi) \mathbf{K}^{\prime}(x), \quad \hat{\boldsymbol{n}} \equiv \widetilde{R} \hat{\boldsymbol{z}},
$$

which is the form of the $\mathbb{Z}_{2}$ symmetry in the new basis. 
Henceforth, we drop the primed superscripts. The most general transformation in $K$ space that corresponds to the presence of the $\mathbb{Z}_{2}$ symmetry in some basis is given by:

$$
\mathbf{K}(x) \rightarrow R(\hat{\boldsymbol{n}}, \pi) \mathbf{K}(x),
$$

where $R(\hat{\boldsymbol{n}}, \pi)$ is a rotation by $180^{\circ}$ about an axis that is parallel to the unit vector $\hat{\boldsymbol{n}}$. As noted in Appendix A, $R(\hat{\boldsymbol{n}}, \pi)$ possesses one non-degenerate eigenvalue equal to +1 and two degenerate eigenvalues -1 . The eigenvector corresponding to the non-degenerate eigenvalue +1 is identified as the rotation axis $\hat{\boldsymbol{n}}$, since

$$
R(\hat{\boldsymbol{n}}, \pi) \hat{\boldsymbol{n}}=\hat{\boldsymbol{n}}
$$

is just the geometrical statement that the rotation axis is unaffected by the rotation. Under the symmetry governed by eq. (5.12), the THDM parameters specified in eq. (2.12) must satisfy eq. (2.13) with $R(U)$ replaced by $R(\hat{\boldsymbol{n}}, \pi)$, which yields:

$$
\begin{aligned}
\boldsymbol{\xi} \text { and } \boldsymbol{\eta} \text { are eigenvectors of } R(\hat{\boldsymbol{n}}, \pi) \text { with eigenvalue }+1 & \Longleftrightarrow \boldsymbol{\xi} \text { and } \boldsymbol{\eta} \text { are parallel to } \hat{\boldsymbol{n}}, \\
E R(\hat{\boldsymbol{n}}, \pi)=R(\hat{\boldsymbol{n}}, \pi) E & \Longleftrightarrow E \hat{\boldsymbol{n}} \text { is parallel to } \hat{\boldsymbol{n}} .
\end{aligned}
$$

Eq. (5.14) is a consequence of the fact the eigenvalue +1 of $R(\hat{\boldsymbol{n}}, \pi)$ is non-degenerate, which implies that any vector $\boldsymbol{v}$ that satisfies $R(\hat{\boldsymbol{n}}, \pi) \boldsymbol{v}=\boldsymbol{v}$ must be proportional to $\hat{\boldsymbol{n}}$. To derive eq. (5.15), we note that $\hat{\boldsymbol{n}}$ is a simultaneous eigenvector of $R(\hat{\boldsymbol{n}}, \pi)$ and $E$. In particular, $R(\hat{\boldsymbol{n}}, \pi) E \hat{\boldsymbol{n}}=E R(\hat{\boldsymbol{n}}, \pi) \hat{\boldsymbol{n}}=E \hat{\boldsymbol{n}}$, where the last step follows from eq. (5.13). Hence either $E \hat{\boldsymbol{n}}=0$ or $E \hat{\boldsymbol{n}}$ is an eigenvector of $R(\hat{\boldsymbol{n}}, \pi)$ with eigenvalue +1 . Since the latter is nondegenerate, it follows that ${ }^{10}$

$$
E_{i j} n_{j} \propto n_{i} .
$$

One can easily check that eqs. (5.14) and (5.16) reduce to eq. (5.5) or eq. (5.9) when $\hat{\boldsymbol{n}}=\hat{\boldsymbol{z}}$ or $\hat{\boldsymbol{x}}$, respectively.

Eq. (5.15) implies that the eigenvectors of $E$ can be chosen to be simultaneous eigenvectors of $R(\hat{\boldsymbol{n}}, \pi)$. Since $E$ is a real symmetric matrix, these eigenvectors can be chosen to be orthonormal. We denote these eigenvectors by $\{\hat{\boldsymbol{n}}, \hat{\boldsymbol{m}}, \hat{\boldsymbol{n}} \times \hat{\boldsymbol{m}}\}$. We have already noted that $\hat{\boldsymbol{n}}$ is an eigenvector of $E$ by virtue of eq. (5.16). Thus, the other two eigenvectors of $E$ must satisfy: ${ }^{11}$

$$
R(\hat{\boldsymbol{n}}, \pi) \hat{\boldsymbol{m}}=-\hat{\boldsymbol{m}}, \quad R(\hat{\boldsymbol{n}}, \pi)(\hat{\boldsymbol{n}} \times \hat{\boldsymbol{m}})=-\hat{\boldsymbol{n}} \times \hat{\boldsymbol{m}} .
$$

Because of the two-fold degeneracy of the eigenvalue -1 of $R(\hat{\boldsymbol{n}}, \pi)$, it is possible to perform orthogonal transformations within the two-dimensional subspace spanned by $\hat{\boldsymbol{m}}$ and $\hat{\boldsymbol{n}} \times \hat{\boldsymbol{m}}$ that leave the form of the $\mathbb{Z}_{2}$ symmetry transformation given in eq. (5.12) unchanged. This simply means that the form of the $\mathbb{Z}_{2}$ symmetry transformation does not uniquely specify the basis in $K$ space. To fix the basis completely, one must specify $\hat{\boldsymbol{n}}$ and the eigenvectors of $R(\hat{\boldsymbol{n}}, \pi)$ corresponding to the two-fold degenerate eigenvalue -1 .

In summary, the $\mathbb{Z}_{2}$ symmetry corresponds to $\mathbf{K}(x) \rightarrow R(\hat{\boldsymbol{n}}, \pi) \mathbf{K}(x)$ for some choice of $\hat{\boldsymbol{n}}$. Imposing this symmetry on the scalar potential requires that

$\boldsymbol{\xi}$ and $\boldsymbol{\eta}$ are parallel to $\hat{\boldsymbol{n}}$,

$E$ is diagonal with respect to the basis $\{\hat{\boldsymbol{n}}, \hat{\boldsymbol{m}}, \hat{\boldsymbol{n}} \times \hat{\boldsymbol{m}}\}$,

where $\hat{\boldsymbol{m}}$ is a simultaneous eigenvector of $R(\hat{\boldsymbol{n}}, \pi)$ and $E$, with $R(\hat{\boldsymbol{n}}, \pi) \hat{\boldsymbol{m}}=-\hat{\boldsymbol{m}}$. In this case, the choice of $\hat{\boldsymbol{n}}$ and $\hat{\boldsymbol{m}}$ uniquely fixes the basis in $K$ space.

\footnotetext{
10 One can derive eq. (5.16) more explicitly by employing eq. A.6) in $E R(\hat{\boldsymbol{n}}, \pi)=R(\hat{\boldsymbol{n}}, \pi) E$. It follows that $n_{i} n_{j} E_{j k}=n_{k} n_{j} E_{j i}$. Using the fact that $E^{\mathrm{T}}=E$, one obtains $E_{i j} n_{j}=C n_{i}$, where the constant of proportionality is identified as $C \equiv n_{j} E_{j k} n_{k}$.

${ }^{11}$ In general, the eigenvectors $\hat{\boldsymbol{m}}_{1}$ and $\hat{\boldsymbol{m}}_{2}$ defined in eq. A.15) are not expected to be eigenvectors of $E$. In this case, $\hat{\boldsymbol{m}}$ is some linear combination of $\hat{\boldsymbol{m}}_{1}$ and $\hat{\boldsymbol{m}}_{2}$, and similarly for $\hat{\boldsymbol{n}} \times \hat{\boldsymbol{m}}$. In particular, $E$ is not generally diagonal with respect to the basis $\left\{\hat{\boldsymbol{n}}, \hat{\boldsymbol{m}}_{1}, \hat{\boldsymbol{m}}_{2}\right\}$.
} 


\section{- U(1) Peccei-Quinn symmetry}

The U(1) Peccei-Quinn symmetry [5, [6, $[$ 8] requires invariance under

$$
\begin{aligned}
& \varphi_{1}(x) \rightarrow e^{-i \theta} \varphi_{1}(x), \\
& \varphi_{2}(x) \rightarrow e^{i \theta} \varphi_{2}(x),
\end{aligned}
$$

with an arbitrary angle $\theta$, which is defined modulo $\pi$. By taking $U=\mathbb{1}_{2} \cos \theta-i \sigma^{3} \sin \theta$ in eq. (2.9), we obtain the corresponding symmetry transformation in $K$ space,

$$
\mathbf{K}(x) \rightarrow R_{2 \theta} \mathbf{K}(x),
$$

where $R_{2 \theta}$ is as in eq. (4.6) with $\alpha=2 \theta$, with $0 \leq \theta<\pi$. That is,

$$
R_{\mathrm{U}(1)}=R_{2 \theta}=\left(\begin{array}{ccc}
\cos 2 \theta & -\sin 2 \theta & 0 \\
\sin 2 \theta & \cos 2 \theta & 0 \\
0 & 0 & 1
\end{array}\right) .
$$

The application of Theorem 1 gives the decomposition [cf. eq. (4.8)]:

$$
R_{2 \theta}=\bar{R}_{2 \theta} \bar{R}_{\theta} .
$$

Requiring the U(1) Peccei-Quinn transformation to be a symmetry implies that the THDM parameters specified in eq. (2.12) must satisfy eq. (2.13) with $R(U)$ replaced by $R_{2 \theta}$ (for all possible values of $\theta$ ), which yields:

$$
\xi_{1}=\xi_{2}=0, \quad \eta_{1}=\eta_{2}=0, \quad E=\operatorname{diag}\left(\mu_{1}, \mu_{1}, \mu_{3}\right) .
$$

It is straightforward to check that imposing the two CP1 symmetries $\bar{R}_{\theta}$ and $\bar{R}_{2 \theta}$ separately is equivalent to requiring invariance under $R_{2 \theta}$.

Remarkably, it is sufficient to require invariance of the scalar potential given by eq. (2.2) under $R_{2 \theta_{0}}$ for any single particular value of $2 \theta_{0} \neq 0(\bmod \pi)[\underline{64}] .{ }^{12}$ That is, invariance under $R_{2 \theta_{0}}$ for any $2 \theta_{0} \neq 0(\bmod \pi)$ implies eq. (5.24) which in turn implies invariance under $R_{2 \theta}$ for all values of $\theta$.

More generally, we consider the possibility that a basis transformation is required to identify the symmetry specified in eq. (5.20). First, we shall rename $2 \theta$ by $\theta$ and rewrite eq. (5.21) as

$$
\mathbf{K}(x) \rightarrow R( \pm \hat{\boldsymbol{z}}, \theta) \mathbf{K}(x),
$$

where $0 \leq \theta \leq \pi$. Note that we must allow for both signs of $\pm \hat{\boldsymbol{z}}$ in order to cover the entire U(1) Peccei-Quinn group manifold. Under a basis transformation specified by the $\mathrm{U}(2)$ matrix $U$ [cf. eqs. (2.5) and (2.6)], we define $\widetilde{R} \equiv R(U)$, where $R(U)$ is given by eq. (2.9). Then, with assistance from eq. (A.20), the U(1) Peccei-Quinn symmetry transformation, eq. (5.25), is transformed to

$$
\mathbf{K}^{\prime}(x) \rightarrow \widetilde{R} R( \pm \hat{\boldsymbol{z}}, \theta) \widetilde{R}^{\mathrm{T}} \mathbf{K}^{\prime}(x)=R( \pm \hat{\boldsymbol{n}}, \theta) \mathbf{K}^{\prime}(x),
$$

where $\hat{\boldsymbol{n}}=\widetilde{R} \hat{\boldsymbol{z}}$ and $R(\hat{\boldsymbol{n}}, \theta)$ is a rotation by $\theta$ about the axis $\hat{\boldsymbol{n}}$.

Thus, dropping the primed subscripts, the most general transformation in $K$ space that corresponds to the presence of the $\mathrm{U}(1)$ Peccei-Quinn symmetry in some basis is given by:

$$
\mathbf{K}(x) \rightarrow R(\hat{\boldsymbol{n}}, \theta) \mathbf{K}(x), \quad \text { for } 0<\theta<\pi .
$$

\footnotetext{
${ }^{12}$ Note that if $\theta_{0}=\pi / 2$ then $R_{2 \theta_{0}}=R_{\pi}$, which generates the $\mathbb{Z}_{2}$ symmetry class treated previously. What is perhaps more surprising is that if $\theta_{0}=\pi / n$ for any integer $n>2$, then the invariance of the scalar potential under $R_{2 \theta_{0}}$ [which generates a $\mathbb{Z}_{n}$ subgroup of U(1)] implies invariance under the full $\mathrm{U}(1)$ group. This latter result is a consequence of the fact that the scalar potential of eq. (2.2) contains no terms of dimension greater than four. If one relaxes this condition, then new symmetry classes can arise that are associated with discrete subgroups of $\mathrm{U}(1)$ of order $d>2$.
} 
Note that we have excluded the case of $\theta=0$, which corresponds to the identity transformation, and the case of $\theta=\pi$, which corresponds to the $\mathbb{Z}_{2}$ symmetry transformation treated previously. When $\theta \neq 0(\bmod \pi), R(\hat{\boldsymbol{n}}, \theta)$ possesses three non-degenerate eigenvalues: $+1, e^{i \theta}$ and $e^{-i \theta}$, and $\hat{\boldsymbol{n}}$ is the normalized eigenvector of $R(\hat{\boldsymbol{n}}, \theta)$ with eigenvalue +1 . It is convenient to introduce normalized eigenvectors $\hat{\boldsymbol{m}}$ and $\hat{\boldsymbol{m}}^{*}$, corresponding to the eigenvalues $e^{i \theta}$ and $e^{-i \theta}$, respectively. For further details, see Appendix A.

Under the symmetry governed by eq. (5.27), the THDM parameters specified in eq. (2.12) must satisfy eq. (2.13) with $R(U)$ replaced by $R(\hat{\boldsymbol{n}}, \theta)$, which yields:

$\boldsymbol{\xi}$ and $\boldsymbol{\eta}$ are eigenvectors of $R(\hat{\boldsymbol{n}}, \theta)$ with eigenvalue $+1 \quad \Longleftrightarrow \quad \boldsymbol{\xi}$ and $\boldsymbol{\eta}$ are parallel to $\hat{\boldsymbol{n}}$,

$$
E R(\hat{\boldsymbol{n}}, \theta)=R(\hat{\boldsymbol{n}}, \theta) E \quad \Longleftrightarrow \quad E \hat{\boldsymbol{v}} \text { is parallel to } \hat{\boldsymbol{v}} \text { for } \hat{\boldsymbol{v}}=\hat{\boldsymbol{n}}, \hat{\boldsymbol{m}}, \hat{\boldsymbol{m}}^{*}
$$

The derivation of eq. (5.29) is similar to the one given in the case of the $\mathbb{Z}_{2}$ symmetry above. We thereby obtain two conditions on the matrix $E$. The first condition,

$$
E_{i j} n_{j} \propto n_{i}
$$

coincides with eq. (5.16). To derive the second condition, we note that $R(\hat{\boldsymbol{n}}, \theta) E \hat{\boldsymbol{m}}=E R(\hat{\boldsymbol{n}}, \theta) \hat{\boldsymbol{m}}=e^{i \theta} E \hat{\boldsymbol{m}}$, which implies that either $E \hat{\boldsymbol{m}}=0$ or $E \hat{\boldsymbol{m}}$ is an eigenvector of $R(\hat{\boldsymbol{n}}, \theta)$ with eigenvalue $e^{i \theta}$. Since $\theta \neq 0(\bmod \pi)$ by assumption, the latter is non-degenerate, and it follows that ${ }^{13}$

$$
E_{i j} m_{j} \propto m_{i}
$$

This equation and its complex conjugate imply that $\hat{\boldsymbol{m}}$ and $\hat{\boldsymbol{m}}^{*}$ are eigenvectors of $E$, whose eigenvalues are complex conjugates of each other. But $E$ is a real symmetric matrix, which implies that its eigenvalues are real. It follows that there is (at least) a two-fold degeneracy among the eigenvalues of $E$.

As noted below eq. (A.14), the eigenvector $\hat{\boldsymbol{m}}$ is independent of the rotation angle $\theta$, assuming that $\theta \neq 0(\bmod \pi)$. Consequently, the constraints on the scalar potential parameters [governed by eqs. (5.28), (5.30) and (5.31)] do not depend on $\theta$. That is, the invariance of the scalar potential under $R(\hat{\boldsymbol{n}}, \theta)$ for any single particular value of $\theta \neq 0$ $(\bmod \pi)$ yields the U(1) Peccei-Quinn symmetry, which in turn implies the invariance of the scalar potential under $R(\hat{\boldsymbol{n}}, \theta)$ for all values of $\theta$.

One can check that eqs. (5.28), (5.30) and (5.31) reduce to eq. (5.24) in the basis where $\hat{\boldsymbol{n}}=\hat{\boldsymbol{z}}$ and $\hat{\boldsymbol{m}}=\frac{1}{\sqrt{2}}(\hat{\boldsymbol{x}}-i \hat{\boldsymbol{y}})$. For example, after inserting $\hat{\boldsymbol{m}}$ into eq. (5.31), and taking into account that $E$ is a real symmetric matrix, we obtain:

$$
E_{11}-i E_{12}=i E_{12}+E_{22}, \quad E_{13}-i E_{23}=0 .
$$

Taking the real and imaginary parts of the above equations, it follows that $E$ is a diagonal matrix with $E_{11}=E_{22}$ as stated in eq. (5.24). Indeed, the above computation is valid for any choice of $\theta \neq 0(\bmod \pi)$, as noted above. Due to eqs. (5.30) and (5.31), the vectors comprising the orthonormal set $\left\{\hat{\boldsymbol{n}}, \hat{\boldsymbol{m}}, \hat{\boldsymbol{m}}^{*}\right\}$ are simultaneous eigenvectors of $R(\hat{\boldsymbol{n}}, \theta)$ and $E$. This means that $E$ is diagonal with respect to the basis $\left\{\hat{\boldsymbol{n}}, \hat{\boldsymbol{m}}, \hat{\boldsymbol{m}}^{*}\right\}$. Since $\hat{\boldsymbol{m}}$ and $\hat{\boldsymbol{m}}^{*}$ are uniquely determined by $\hat{\boldsymbol{n}}$ [cf. eqs. (A.13) and (A.14)] when $\theta \neq 0(\bmod \pi)$, it follows that $\hat{\boldsymbol{n}}$ uniquely fixes the basis in $K$ space.

\section{- CP1 symmetry}

In Section 3, we showed that one can always find a basis in which the CP1 transformation in $K$ space is given by

$$
\mathbf{K}(x) \rightarrow \bar{R}_{2} \mathbf{K}\left(x^{\prime}\right)
$$

13 One can derive eq. (5.31) more explicitly by employing eq. A.5 in $E R(\hat{\boldsymbol{n}}, \theta)=R(\hat{\boldsymbol{n}}, \theta) E$. This yields two constrains: $n_{i} n_{j} E_{j k}=n_{k} n_{j} E_{j i}$ (also obtained in the case of the $\mathbb{Z}_{2}$ symmetry, as noted in footnote 10 ) and $\epsilon_{i j \ell} E_{j k} n_{\ell}=\epsilon_{j k \ell} E_{i j} n_{\ell}$. If we multiply the latter equation by $m_{k}$ and employ $E^{\mathrm{T}}=E, E \hat{\boldsymbol{n}} \propto \hat{\boldsymbol{n}}$ and $\hat{\boldsymbol{n}} \cdot \hat{\boldsymbol{m}}=0$, it then follows that $E_{i j} m_{j}=\kappa m_{i}$, where the constant of proportionality is identified as $\kappa \equiv \frac{1}{2}\left(\operatorname{Tr} E-n_{i} E_{i j} n_{j}\right)$. 
That is,

$$
\bar{R}_{\mathrm{CP} 1}=\bar{R}_{2}=\left(\begin{array}{rrr}
1 & 0 & 0 \\
0 & -1 & 0 \\
0 & 0 & 1
\end{array}\right) .
$$

Requiring CP1 to be a symmetry implies that the THDM parameters specified in eq. (2.12) must satisfy eq. (3.40) with $\bar{R} \equiv \bar{R}_{\mathrm{CP} 1}$, which yields:

$$
\xi_{2}=0, \eta_{2}=0, E_{12}=E_{23}=0 .
$$

As expected, eq. (5.35) is equivalent to the statement that in a basis in which the CP1 transformation is given by eq. (5.33), all the parameters of the scalar potential specified in eq. (2.2) are real.

As in the case of the $\mathbb{Z}_{2}$ symmetry, there still remains some freedom to perform a basis transformation while maintaining the form of the CP1 symmetry given in eq. (5.33). By performing a change of basis characterized by an $\mathrm{SO}(3)$ matrix $R$ with $R_{12}=R_{23}=R_{21}=R_{32}=0$ and $R_{22}=1$, we see that eq. (5.35) is still satisfied, while other matrix elements of $E$ are transformed according to $E \rightarrow R E R^{\mathrm{T}}$. One is free to choose $R$ such that $E$ in the new basis is diagonal.

The above results pertain to a specific basis choice. With respect to an arbitrary basis, we showed in Section 3 that $\bar{R}_{\mathrm{CP} 1}$ is a reflection through planes in $K$ space, which implies that $\bar{R}_{\mathrm{CP} 1}$ is an improper rotation matrix that satisfies

$$
\bar{R}_{\mathrm{CP} 1}^{2}=\mathbb{1}_{3}, \quad \bar{R}_{\mathrm{CP} 1} \neq-\mathbb{1}_{3} .
$$

An explicit form is given by:

$$
\bar{R}_{\mathrm{CP} 1}=-R(\hat{\boldsymbol{n}}, \pi)
$$

where the unit vector $\hat{\boldsymbol{n}}$ points in the direction normal to the reflection plane. In general, $-R(\hat{\boldsymbol{n}}, \pi)$ possesses one non-degenerate eigenvalue -1 and a doubly-degenerate eigenvalue +1 . We identify $\hat{\boldsymbol{n}}$ as the eigenvector of $-R(\hat{\boldsymbol{n}}, \pi)$ corresponding to the non-degenerate eigenvector -1 . The eigenvectors corresponding to the doubly-degenerate eigenvalues of $\bar{R}_{\mathrm{CP} 1}$ span the reflection plane in $K$ space.

The scalar potential exhibits the CP1 symmetry, eq. (5.33), in some basis if

$$
\mathbf{K}(x) \rightarrow-R(\hat{\boldsymbol{n}}, \pi) \mathbf{K}\left(x^{\prime}\right)
$$

is a symmetry. That is, the THDM parameters specified in eq. (2.12) must satisfy eq. (3.40) with $\bar{R} \equiv \bar{R}_{\mathrm{CP} 1}$, which yields:

$$
\begin{aligned}
\boldsymbol{\xi} \text { and } \boldsymbol{\eta} \text { are eigenvectors of }-R(\hat{\boldsymbol{n}}, \pi) \text { with eigenvalue }+1 & \Longleftrightarrow \boldsymbol{\xi} \text { and } \boldsymbol{\eta} \text { are perpendicular to } \hat{\boldsymbol{n}}, \\
E R(\hat{\boldsymbol{n}}, \pi)=R(\hat{\boldsymbol{n}}, \pi) E & \Longleftrightarrow E \hat{\boldsymbol{n}} \text { is parallel to } \hat{\boldsymbol{n}}
\end{aligned}
$$

Following the derivation of eq. (5.16), either $E \hat{\boldsymbol{n}}=0$ or $E \hat{\boldsymbol{n}}$ is an eigenvector of $-R(\hat{\boldsymbol{n}}, \pi)$ with eigenvalue -1 . Since the latter is non-degenerate, we again recover eq. (5.16). It is a simple matter to confirm that eqs. (5.16) and (5.39) reduce to eq. (5.35) when $\hat{\boldsymbol{n}}=\hat{\boldsymbol{y}}$.

We can introduce simultaneous eigenvectors of $E$ and $-R(\hat{\boldsymbol{n}}, \pi)$, denoted by $\hat{\boldsymbol{m}}$ and $\hat{\boldsymbol{n}} \times \hat{\boldsymbol{m}}$, which satisfy eq. (5.17) [cf. footnote 11]. These two vectors correspond to the two-fold degenerate eigenvalue +1 of $-R(\hat{\boldsymbol{n}}, \pi)$. As in the treatment of the $\mathbb{Z}_{2}$ symmetry, the form of the CP1 symmetry transformation does not uniquely specify the basis in $K$ space. To fix the basis completely, one must specify $\hat{\boldsymbol{n}}$ and the eigenvectors of $-R(\hat{\boldsymbol{n}}, \pi)$ corresponding to the two-fold degenerate eigenvalue +1 .

In summary, the CP1 symmetry corresponds to $\mathbf{K}(x) \rightarrow-R(\hat{\boldsymbol{n}}, \pi) \mathbf{K}\left(x^{\prime}\right)$ for some choice of $\hat{\boldsymbol{n}}$. Imposing this symmetry on the scalar potential requires that

$\boldsymbol{\xi}$ and $\boldsymbol{\eta}$ are perpendicular to $\hat{\boldsymbol{n}}$,

$E$ is diagonal with respect to the basis $\{\hat{\boldsymbol{n}}, \hat{\boldsymbol{m}}, \hat{\boldsymbol{n}} \times \hat{\boldsymbol{m}}\}$, 
where $\hat{\boldsymbol{m}}$ is a simultaneous eigenvector of $-R(\hat{\boldsymbol{n}}, \pi)$ and $E$, with $-R(\hat{\boldsymbol{n}}, \pi) \hat{\boldsymbol{m}}=\hat{\boldsymbol{m}}$. In this case, the choice of $\hat{\boldsymbol{n}}$ and $\hat{\boldsymbol{m}}$ uniquely fixes the basis in $K$ space.

\section{- CP2 symmetry}

The CP2 symmetry class is generated by requiring that the scalar potential parameters specified in eq. (2.12) are invariant under $\bar{R}=-R(\hat{\boldsymbol{n}}, 0)=-\mathbb{1}_{3}$ corresponding to $2 \theta=\pi$ in eq. (4.10), which yields

$$
\bar{R}_{\mathrm{CP} 2}=\bar{R}_{\pi}^{\prime}=\left(\begin{array}{rrr}
-1 & 0 & 0 \\
0 & -1 & 0 \\
0 & 0 & -1
\end{array}\right) .
$$

The form of $\bar{R}_{\mathrm{CP} 2}$ is basis-independent, since $R(U) \bar{R}_{\mathrm{CP} 2} R^{\mathrm{T}}(U)=\bar{R}_{\mathrm{CP} 2}$ for any choice of $U$. We may now invoke Theorem 2 from Section 4. From eq. (4.11), we then obtain

$$
\bar{R}_{3} \bar{R}_{2} \bar{R}_{1}=-\mathbb{1}_{3}
$$

Invariance under $\bar{R}_{\mathrm{CP} 2}=-\mathbb{1}_{3}$ requires $\boldsymbol{\xi}=0$ and $\boldsymbol{\eta}=0$ but leaves $E$ arbitrary. We can then perform a basis transformation to diagonalize $E$, which leaves $\boldsymbol{\xi}$ and $\boldsymbol{\eta}$ unchanged. Invariance under all three individual CP1 transformations $\bar{R}_{3}, \bar{R}_{2}$, and $\bar{R}_{1}$, taken together, implies that

$$
\boldsymbol{\xi}=\boldsymbol{\eta}=0 \quad \text { and } \quad E=\operatorname{diag}\left(\mu_{1}, \mu_{2}, \mu_{3}\right) .
$$

Clearly, $\bar{R}_{3}, \bar{R}_{2}$, and $\bar{R}_{1}$, when separately applied, yield a stronger constraint than $-\mathbb{1}_{3}$ alone. But the three CP1 symmetries are physically equivalent to $-\mathbb{1}_{3}$, since the scalar potentials that result from the two procedures are related by the change of basis that diagonalizes $E$. As shown in [46], this equivalence may no longer hold once the Yukawa sector is taken into account.

\section{- CP3 symmetry}

It is convenient to choose a basis in which the CP3 symmetry class is generated as follows. We require the transformation $\bar{R}_{2 \theta_{1}}^{\prime}$ [defined in eq. (4.10)] to be a symmetry, for any choice of angle such that $2 \theta_{1} \neq 0(\bmod \pi)$. Theorem 2 can again be employed to express $\bar{R}_{2 \theta_{1}}^{\prime}$ as a product of three CP1 transformations. Eqs. (4.10) and (4.11) then yield:

$$
\bar{R}_{\mathrm{CP} 3}=\bar{R}_{2 \theta_{1}}^{\prime}=\left(\begin{array}{ccr}
\cos 2 \theta_{1} & -\sin 2 \theta_{1} & 0 \\
\sin 2 \theta_{1} & \cos 2 \theta_{1} & 0 \\
0 & 0 & -1
\end{array}\right)=\bar{R}_{3} \bar{R}_{2 \theta_{1}} \bar{R}_{\theta_{1}}
$$

Requiring CP3 to be a symmetry implies that the THDM parameters specified in eq. (2.12) must satisfy eq. (3.40) with $\bar{R} \equiv \bar{R}_{2 \theta_{1}}^{\prime}$, which yields:

$$
\boldsymbol{\xi}=\boldsymbol{\eta}=0, \quad E=\operatorname{diag}\left(\mu_{1}, \mu_{1}, \mu_{3}\right) .
$$

It is straightforward to check that imposing the three CP1 symmetries $\bar{R}_{3}, \bar{R}_{2 \theta_{1}}$ and $\bar{R}_{\theta_{1}}$ separately is equivalent to requiring invariance under $\bar{R}_{2 \theta_{1}}^{\prime}$. Similarly to the case of the U(1) Peccei-Quinn symmetry, the invariance of the scalar potential under $\bar{R}_{2 \theta_{1}}^{\prime}$, for any single particular value of $2 \theta_{1} \neq 0(\bmod \pi)$, implies invariance under $\bar{R}_{2 \theta}^{\prime}$ for all values of $\theta$.

The above results pertain to a specific basis choice. With respect to an arbitrary basis, we showed in Section 3 that $\bar{R}_{\mathrm{CP} 3}$ is an improper rotation matrix that satisfies $\bar{R}_{\mathrm{CP} 3}^{2} \neq \mathbb{1}_{3}$ (i.e., $\bar{R}_{\mathrm{CP} 3}$ is not a pure reflection in $K$ space). The 
most general form for the CP3 transformation is given by $\bar{R}_{\mathrm{CP} 3}=-R(\hat{\boldsymbol{n}}, \theta)$ for $0<\theta<\pi$. Thus the scalar potential, in a basis specified by $\hat{\boldsymbol{n}}$, exhibits the CP3 symmetry if it is invariant under the transformation

$$
\mathbf{K}(x) \rightarrow-R(\hat{\boldsymbol{n}}, \theta) \mathbf{K}\left(x^{\prime}\right), \quad \text { for } 0<\theta<\pi .
$$

Note that we have excluded the case of $\theta=0$, which corresponds to the CP2 symmetry transformation, and the case of $\theta=\pi$, which corresponds to the CP1 symmetry transformation, both of which have already been treated above. When $\theta \neq 0(\bmod \pi)$, the improper rotation matrix $-\bar{R}(\hat{\boldsymbol{n}}, \theta)$ possesses three non-degenerate eigenvalues: -1 , $-e^{i \theta}$ and $-e^{-i \theta}$, and $\hat{\boldsymbol{n}}$ is the normalized eigenvector of $-R(\hat{\boldsymbol{n}}, \theta)$ with eigenvalue -1 . As in the analysis of the $\mathrm{U}(1)$ Peccei-Quinn symmetry, it is convenient to introduce normalized eigenvectors of $-R(\hat{\boldsymbol{n}}, \theta)$, denoted by $\hat{\boldsymbol{m}}$ and $\hat{\boldsymbol{m}}^{*}$, corresponding to the eigenvalues $-e^{i \theta}$ and $-e^{-i \theta}$, respectively.

Under the symmetry governed by eq. (5.48), the THDM parameters specified in eq. (2.12) must satisfy eq. (3.40) with $\bar{R} \equiv-R(\hat{\boldsymbol{n}}, \theta)$. Since $-R(\hat{\boldsymbol{n}}, \theta)$ has no eigenvalue +1 for $\theta \neq 0(\bmod \pi)$, it follows that:

$$
\begin{aligned}
-R(\hat{\boldsymbol{n}}, \theta) \boldsymbol{\xi}=\boldsymbol{\xi}, \quad-R(\hat{\boldsymbol{n}}, \theta) \boldsymbol{\eta}=\boldsymbol{\eta}, \quad \Longleftrightarrow \quad \boldsymbol{\xi}=\boldsymbol{\eta}=0, \\
E R(\hat{\boldsymbol{n}}, \theta)=R(\hat{\boldsymbol{n}}, \theta) E \quad \Longleftrightarrow \quad E \hat{\boldsymbol{v}} \text { is parallel to } \hat{\boldsymbol{v}} \text { for } \hat{\boldsymbol{v}}=\hat{\boldsymbol{n}}, \hat{\boldsymbol{m}}, \hat{\boldsymbol{m}}^{*}
\end{aligned}
$$

The derivation of eq. (5.50) is identical to the one given in the analysis of the U(1) Peccei-Quinn symmetry. Thus, we again recover the results of eqs. (5.30) and (5.31),

$$
E_{i j} n_{j} \propto n_{i} \quad \text { and } \quad E_{i j} m_{j} \propto m_{i} .
$$

For the same reasons given below eq. (5.31), there is (at least) a two-fold degeneracy among the eigenvalues of $E$. Since the constraints imposed by eqs. (5.49) and (5.51) are independent of the angle $\theta$, the invariance of the scalar potential under $-R(\hat{\boldsymbol{n}}, \theta)$ for any value of $\theta \neq 0(\bmod \pi)$ yields the CP3 symmetry, which in turn implies the invariance of the scalar potential under $-R(\hat{\boldsymbol{n}}, \theta)$ for all values of $\theta$. As expected, when $\hat{\boldsymbol{n}}=\hat{\boldsymbol{z}}$ and $\hat{\boldsymbol{m}}=\frac{1}{\sqrt{2}}(\hat{\boldsymbol{x}}-i \hat{\boldsymbol{y}})$, eqs. (5.49) and (5.51) lead to the previous results given in eq. (5.47) for any value of $\theta \neq 0(\bmod \pi)$.

By virtue of eq. (5.51), the vectors comprising the orthonormal set $\left\{\hat{\boldsymbol{n}}, \hat{\boldsymbol{m}}, \hat{\boldsymbol{m}}^{*}\right\}$ are simultaneous eigenvectors of $-R(\hat{\boldsymbol{n}}, \theta)$ and $E$. This means that $E$ is diagonal with respect to the basis $\left\{\hat{\boldsymbol{n}}, \hat{\boldsymbol{m}}, \hat{\boldsymbol{m}}^{*}\right\}$. Since $\hat{\boldsymbol{m}}$ and $\hat{\boldsymbol{m}}^{*}$ are uniquely determined by $\hat{\boldsymbol{n}}$ [cf. eqs. (A.13) and (A.14)] when $\theta \neq 0(\bmod \pi)$, it follows that $\hat{\boldsymbol{n}}$ uniquely fixes the basis in $K$ space.

\section{- The SO(3)-symmetric scalar potential}

Suppose that the THDM scalar potential is invariant under all possible Higgs-family transformations [cf. eq. (2.5)]. The $\mathrm{U}(2)$ invariance group contains a gauged hypercharge $\mathrm{U}(1)$ subgroup, which is automatically satisfied by the Higgs Lagrangian. The ungauged invariance group is therefore isomorphic to $\mathrm{SO}(3)$. The elements of this $\mathrm{SO}(3)$ group are in one-to-one correspondence with the matrices $R(U)$ defined in eq. (2.9). Hence, the invariance of the scalar potential under $\mathrm{SO}(3)$ requires that eq. (2.13) must be satisfied for any $\mathrm{SO}(3)$ matrix $R(U) \in \mathrm{SO}(3)$. This clearly implies that

$$
\boldsymbol{\xi}=0, \quad \boldsymbol{\eta}=0, \quad E=\mu \mathbb{1}_{3},
$$

which of course must hold for any choice of basis (since the set of all basis transformations has been promoted to a global symmetry of the scalar potential). In $K$ space, one rotation matrix is not sufficient to generate all the elements of the $\mathrm{SO}(3)$ symmetry class. Since any rotation matrix must be of the form $R(\hat{\boldsymbol{n}}, \theta)$, the results of Section 5.1 imply that a single rotation matrix will generate either the $\mathbb{Z}_{2}$ symmetry class (if $\theta=\pi$ ) or the U(1) Peccei-Quinn symmetry class, if $0<\theta<\pi$. However, one can generate all the elements of the $\mathrm{SO}(3)$ symmetry class with two rotation matrices, $R\left(\hat{\boldsymbol{n}}_{1}, \theta\right)$ and $R\left(\hat{\boldsymbol{n}}_{2}, \theta\right)$, where $\hat{\boldsymbol{n}}_{1} \times \hat{\boldsymbol{n}}_{2} \neq 0$. In group theory language, this is just the statement that starting from two non-trivial rotations for which the corresponding rotation axes are non-collinear, it is possible to generate any element of $\mathrm{SO}(3)$ by successive multiplication of the two initial rotation matrices and products thereof. 
For example, the $\mathrm{SO}(3)$ symmetry class can be generated through the application of both

$$
R_{\alpha_{1}}=\left(\begin{array}{ccc}
\cos \alpha_{1} & -\sin \alpha_{1} & 0 \\
\sin \alpha_{1} & \cos \alpha_{1} & 0 \\
0 & 0 & 1
\end{array}\right), \quad \text { with } 0<\alpha_{1}<\pi
$$

and

$$
R_{\alpha_{2}}^{\prime}=\left(\begin{array}{ccc}
1 & 0 & 0 \\
0 & \cos \alpha_{2} & -\sin \alpha_{2} \\
0 & \sin \alpha_{2} & \cos \alpha_{2}
\end{array}\right), \quad \text { with } 0<\alpha_{2}<\pi
$$

Since there is a infinite number of axes non-collinear with the axis of the first rotation, we have infinitely many ways to generate $\mathrm{SO}(3)$ through combinations of Higgs-family transformations.

In fact, the maximal invariance group [orthogonal to the gauged hypercharge $\mathrm{U}(1)$ ] of the $\mathrm{SO}(3)$-symmetric scalar potential is $\mathrm{O}(3)$, as noted in [44, 50]. This can be seen either by examining the symmetry transformation properties of the scalar fields or via the transformations of the scalar field bilinears. Eq. (5.52) implies that the scalar potential is invariant with respect to Higgs family transformations, $\varphi_{i}(x) \rightarrow U_{i j} \varphi_{j}(x)$, for any choice of the unitary matrix $U$. Since all potentially complex parameters of the $\mathrm{SO}(3)$-invariant scalar potential are zero, eq. (5.52) also implies that the scalar potential is invariant with respect to GCP transformations, $\varphi_{i}(x) \rightarrow X_{i j} \varphi_{j}^{*}\left(x^{\prime}\right)$, for any choice of the unitary matrix $X$. Mathematically, complex conjugation is isomorphic to the discrete group $\mathbb{Z}_{2}$, and hence the relevant invariance group is $\mathrm{SO}(3) \otimes \mathbb{Z}_{2} \cong \mathrm{O}(3)$. In $K$ space, it immediately follows from eq. (5.52) that eqs. (2.13) and (3.40) are satisfied for all proper and improper rotation matrices $R$ and $\bar{R}$, respectively. In particular, using the results of Section 5.1, we immediately conclude that the $K$ space symmetries of the $\mathrm{SO}(3)$-invariant scalar potential can also be generated by a product of two non-collinear CP3 transformations, $-R\left(\hat{\boldsymbol{n}}_{1}, \theta\right)$ and $-R\left(\hat{\boldsymbol{n}}_{2}, \theta\right)$, or by a product of the Higgs family transformation $R\left(\hat{\boldsymbol{n}}_{1}, \theta\right)$ and a CP3 transformation $-R\left(\hat{\boldsymbol{n}}_{2}, \theta\right)$, where $\hat{\boldsymbol{n}}_{1} \times \hat{\boldsymbol{n}}_{2} \neq 0$. Thus, the full $\mathrm{O}(3)$ group consisting of all proper and improper rotation matrices is generated. ${ }^{14}$

\subsection{Identifying the THDM symmetry classes}

We have examined the constraints on the scalar potential parameters for each of the six symmetry classes listed in Table I. We can reverse the process and determine the symmetry class given the scalar potential parameters. We summarize the results of Section 5.1 in Table II, which agrees, of course, with the basis-independent characterization of the symmetry classes given in [39, 44, 65]. In particular, given a set of constraints on $\boldsymbol{\xi}, \boldsymbol{\eta}$ and the eigenvalues and eigenvectors of $E$, one can uniquely determine the maximal symmetry class of the scalar potential. Note that $\boldsymbol{\xi} \times \boldsymbol{\eta}=0$ for all symmetry classes, with the possible exception of CP1. In the case of CP1, the only necessary conditions on the scalar potential parameters are: ${ }^{15}$

$$
\boldsymbol{\xi} \cdot \hat{\boldsymbol{e}}_{i}=\boldsymbol{\eta} \cdot \hat{\boldsymbol{e}}_{i}=0, \quad(\boldsymbol{\xi}, \boldsymbol{\eta}) \neq(0,0)
$$

where $\hat{\boldsymbol{e}}_{i}$ is one of the three eigenvectors of $E$ (independently of the degeneracy of the eigenvalues of $E$ ). When the scalar potential parameters are generic, $\boldsymbol{\xi} \times \boldsymbol{\eta} \neq 0$. In this case the constraints given in eq. (5.55) are equivalent to the statement that $\boldsymbol{\xi} \times \boldsymbol{\eta}$ is an eigenvector of $E[39] .{ }^{16}$ On the other hand, if $\boldsymbol{\xi} \times \boldsymbol{\eta}=0$, then the symmetry class

\footnotetext{
14 The reader is cautioned that after the inclusion of the Yukawa sector, the corresponding symmetry properties of each of these pairs of transformations might be different from the pure scalar theory.

15 Using the results of Table II, it is easy to check that $\boldsymbol{\xi} \cdot \hat{\boldsymbol{e}}=\boldsymbol{\eta} \cdot \hat{\boldsymbol{e}}=0$ (where $\hat{\boldsymbol{e}}$ is one of the eigenvectors of $E$ ) must hold for all symmetry classes. For the higher symmetry classes, additional constraints must be satisfied as exhibited in Table II.

16 In this case, one can define $\hat{\boldsymbol{n}}$ as the unit vector that points in the direction of $\boldsymbol{\xi} \times \boldsymbol{\eta}$, and eq. (5.41) is automatically satisfied.
} 


\begin{tabular}{|c|c|c|}
\hline symmetry class & constraints on $\boldsymbol{\xi}$ and $\boldsymbol{\eta}$ & constraints on $E$ \\
\hline \multirow[t]{3}{*}{$\mathbb{Z}_{2}$} & $\overline{\boldsymbol{\xi}} \times \hat{\boldsymbol{e}}=\boldsymbol{\eta} \times \hat{\boldsymbol{e}}=0 ;(\boldsymbol{\xi}, \boldsymbol{\eta}) \neq(0,0)$ & eigenvalues are non-degenerate \\
\hline & or & or \\
\hline & $\boldsymbol{\xi} \times \hat{\boldsymbol{e}}^{\prime}=\boldsymbol{\eta} \times \hat{\boldsymbol{e}}^{\prime}=0 ;(\boldsymbol{\xi}, \boldsymbol{\eta}) \neq(0,0)$ & $E$ possesses doubly-degenerate eigenvalues \\
\hline \multirow[t]{3}{*}{$\mathrm{U}(1)$} & $\boldsymbol{\xi} \times \hat{\boldsymbol{e}}=\boldsymbol{\eta} \times \hat{\boldsymbol{e}}=0 ;(\boldsymbol{\xi}, \boldsymbol{\eta}) \neq(0,0)$ & $E$ possesses doubly-degenerate eigenvalues \\
\hline & or & or \\
\hline & $\boldsymbol{\xi} \times \boldsymbol{\eta}=0 ;(\boldsymbol{\xi}, \boldsymbol{\eta}) \neq(0,0)$ & $E$ possesses triply-degenerate eigenvalues $\left(E=\mu \mathbb{1}_{3}\right)$ \\
\hline $\mathrm{SO}(3)$ & $(\boldsymbol{\xi}, \boldsymbol{\eta})=(0,0)$ & $E$ possesses triply-degenerate eigenvalues $\left(E=\mu \mathbb{1}_{3}\right)$ \\
\hline \multirow[t]{5}{*}{$\mathrm{CP} 1$} & $\boldsymbol{\xi} \times \boldsymbol{\eta}$ is an eigenvector of $E$ & eigenvalues are unconstrained \\
\hline & or & or \\
\hline & $\begin{array}{l}\boldsymbol{\xi} \times \boldsymbol{\eta}=0 ; \boldsymbol{\xi} \cdot \hat{\boldsymbol{e}}=\boldsymbol{\eta} \cdot \hat{\boldsymbol{e}}=0 ;(\boldsymbol{\xi}, \boldsymbol{\eta}) \neq(0,0) \\
\text { neither } \boldsymbol{\xi} \text { nor } \boldsymbol{\eta} \text { is an eigenvector of } E\end{array}$ & eigenvalues are non-degenerate \\
\hline & or & or \\
\hline & $\begin{array}{l}\boldsymbol{\xi} \times \boldsymbol{\eta}=0 ; \boldsymbol{\xi} \cdot \hat{\boldsymbol{e}}^{\prime}=\boldsymbol{\eta} \cdot \hat{\boldsymbol{e}}^{\prime}=0 ;(\boldsymbol{\xi}, \boldsymbol{\eta}) \neq(0,0) \\
\text { neither } \boldsymbol{\xi} \text { nor } \boldsymbol{\eta} \text { is an eigenvector of } E\end{array}$ & $E$ possesses doubly-degenerate eigenvalues \\
\hline $\mathrm{CP} 2$ & $(\boldsymbol{\xi}, \boldsymbol{\eta})=(0,0)$ & eigenvalues are non-degenerate \\
\hline CP3 & $(\boldsymbol{\xi}, \boldsymbol{\eta})=(0,0)$ & $E$ possesses doubly-degenerate eigenvalues \\
\hline
\end{tabular}

TABLE II: The symmetry classes and the corresponding constraints on the scalar potential parameters. The unit vector $\hat{\boldsymbol{e}}$ is one of the three eigenvectors of $E$ corresponding to a non-degenerate eigenvalue of $E$, and the unit vector $\hat{\boldsymbol{e}}^{\prime}$ is an eigenvector of $E$ corresponding to a doubly-degenerate eigenvalue of $E$. Note that if $(\boldsymbol{\xi}, \boldsymbol{\eta})=(0,0)$, then the eigenvectors of $E$ play no role in constraining the scalar potential parameters. For the U(1) symmetry class, $\hat{e}$ is uniquely identified as the eigenvector of $E$ corresponding to the non-degenerate eigenvalue (if it exists). For the CP1 symmetry class, $\boldsymbol{\xi}$ and $\boldsymbol{\eta}$ are necessarily orthogonal to one of the eigenvectors of $E$. If $\boldsymbol{\xi}$ and $\boldsymbol{\eta}$ are collinear [and $(\boldsymbol{\xi}, \boldsymbol{\eta}) \neq(0,0)$ ], then we must impose one additional condition to ensure that the constraints relevant for the $\mathbb{Z}_{2}$ or $\mathrm{U}(1)$ symmetry classes are not satisfied.

of the scalar potential will be CP1 if and only if the constraints of the other symmetry classes listed in Table II are not satisfied. For example, consider the case where one eigenvalue of $E$ is non-degenerate and corresponds to the eigenvector $\hat{\boldsymbol{e}}_{i}$ in eq. (5.55), and the other eigenvalue of $E$ is doubly-degenerate. Then eq. (5.55) necessarily implies that the collinear vectors $\boldsymbol{\xi}$ and $\boldsymbol{\eta}$ are also collinear with some linear combination of the other two eigenvectors $\hat{\boldsymbol{e}}_{j}$ $(j \neq i)$ of $E$, and the scalar potential therefore exhibits either the $\mathbb{Z}_{2}$ or $\mathrm{U}(1)$ symmetry. If the eigenvalues of $E$ are non-degenerate, or if $\hat{\boldsymbol{e}}_{i}$ in eq. (5.55) corresponds to a doubly-degenerate eigenvalue of $E$, then it is possible that neither $\boldsymbol{\xi}$ nor $\boldsymbol{\eta}$ is an eigenvector of $E$, in which case the symmetry class of the scalar potential is CP1.

Suppose that the scalar potential parameters satisfy the constraints of a particular symmetry class. By adding additional constraints, it is often possible to promote the scalar potential to a class with a larger symmetry. That is, there is a hierarchy of symmetries that can schematically be represented by the following chain:

$$
\mathrm{CP} 1<\mathbb{Z}_{2}<\left\{\begin{array}{l}
\mathrm{U}(1) \\
\mathrm{CP} 2
\end{array}\right\}<\mathrm{CP} 3<\mathrm{SO}(3) .
$$

The meaning of the above equation is the following. A scalar potential that exhibits a $\mathbb{Z}_{2}$ symmetry also must exhibit a CP1 symmetry. If the scalar potential exhibits a U(1) symmetry, then it must also exhibit the symmetries that precede it in the chain, and so on. Note that the U(1) symmetry does not imply a CP2 symmetry and vice versa. However, a CP3-symmetric potential must exhibit both the U(1) symmetry and the CP2 symmetry. Eq. (5.56) is a consequence of the results of Table II For example, if we take a CP2-symmetric scalar potential and impose the additional condition that one of the eigenvalues of $E$ is doubly-degenerate, then we promote the symmetry to CP3. Likewise, if we take a $\mathrm{U}(1)$-symmetric scalar potential (assuming $E \neq \mu \mathbb{1}_{3}$ ) and then impose the additional constraint $\boldsymbol{\xi}=\boldsymbol{\eta}=0$, then we promote the symmetry to CP3. The rest of the hierarchy of symmetries can be deduced in a similar manner.

Note that there are cases in which the symmetry is not enhanced if the degeneracy of one of the eigenvalues of $E$ is increased. Indeed, these cases do not correspond to new symemtry classes beyond the six listed in Table II. This 
conclusion is a consequence of a classification theorem, originally proved in [4] based on a geometrical analysis, ${ }^{17}$ which states that the only possible symmetry classes of the THDM are those listed in Table I. This result can be checked by employing the renormalization group equations (RGEs) for the scalar potential parameters. In particular, the constraints on the scalar potential parameters for a given symmetry class must be respected at any scale and hence invariant under renormalization group evolution.

The RGEs for the scalar potential parameters in the general THDM can be found in [18, 48, 66, 67]. One can check that all the parameter constraints exhibited in Table II are invariant under renormalization group running. However, if there are further accidental degeneracies among the eigenvalues of $E$, these degeneracies will not in general be renormalization group invariant. As a simple example, consider a U(1)-invariant scalar potential in a basis where $m_{12}^{2}=\lambda_{5}=\lambda_{6}=\lambda_{7}=0$. That is, we have from eq. (2.12)

$$
\begin{aligned}
& \eta_{00}=\frac{1}{8}\left(\lambda_{1}+\lambda_{2}\right)+\frac{1}{4} \lambda_{3}, \quad \boldsymbol{\xi}=\left(\begin{array}{c}
0 \\
0 \\
\xi_{3}=\frac{1}{2}\left(m_{11}^{2}-m_{22}^{2}\right)
\end{array}\right), \quad \boldsymbol{\eta}=\left(\begin{array}{c}
0 \\
0 \\
\eta_{3}=\frac{1}{8}\left(\lambda_{1}-\lambda_{2}\right)
\end{array}\right), \\
& E=\frac{1}{4} \operatorname{diag}\left(\lambda_{4}, \lambda_{4}, \frac{1}{2}\left(\lambda_{1}+\lambda_{2}\right)-\lambda_{3}\right) .
\end{aligned}
$$

In this case $\hat{\boldsymbol{e}}=(0,0,1)$, and the constraints listed in Table II are clearly satisfied. Now, suppose we add the following additional constraint: $\lambda_{1}+\lambda_{2}=2\left(\lambda_{3}+\lambda_{4}\right)$. In this case, the matrix $E$ possesses one triply-degenerate eigenvalue, and $E=\mu \mathbb{1}_{3}$ with $\mu \equiv \lambda_{4} / 4$. However, this is not a new symmetry class because the constraint that $\lambda_{1}+\lambda_{2}=2\left(\lambda_{3}+\lambda_{4}\right)$ is not in general renormalization group invariant. Using the RGEs for the scalar potential parameters with $\lambda_{5}=\lambda_{6}=\lambda_{7}=0$, we find:

$$
8 \pi^{2} \beta_{\lambda_{1}+\lambda_{2}-2\left(\lambda_{3}+\lambda_{4}\right)}=\left[\lambda_{1}+\lambda_{2}-2\left(\lambda_{3}+\lambda_{4}\right)\right]\left[3\left(\lambda_{1}+\lambda_{2}\right)+2 \lambda_{4}\right]+3\left(\lambda_{1}-\lambda_{2}\right)^{2} .
$$

Thus, if $\lambda_{1} \neq \lambda_{2}$, then the condition $\lambda_{1}+\lambda_{2}-2\left(\lambda_{3}+\lambda_{4}\right)=0$ is not stable under renormalization group running. If in addition $\lambda_{1}=\lambda_{2}$ and $m_{11}^{2}=m_{22}^{2}$, then the scalar potential is $\mathrm{SO}(3)$-invariant, corresponding to one of the other symmetry classes of Table II. Note that it is sufficient to set $\lambda_{1}=\lambda_{2}$ in eq. (5.58) to obtain a fixed point in the RGE at $\lambda_{1}+\lambda_{2}=2\left(\lambda_{3}+\lambda_{4}\right)=0$. This would correspond to a softly broken $\mathrm{SO}(3)$-invariant scalar potential, where the soft-breaking is due to $m_{11}^{2} \neq m_{22}^{2}$. Of course, this soft symmetry breaking is invisible to the RGE running of the coefficients of dimension-four terms of the Higgs Lagrangian.

In general, additional degeneracies in the eigenvalues of $E$ in Table II are either unstable with respect to renormalization group running or else correspond to a known symmetry class of enhanced symmetry that is either exact or softly-broken by dimension-two terms of the scalar potential. Thus, we conclude that no additional symmetry classes beyond those listed in Table II exist, in agreement with the classification theorem of [44].

\subsection{Symmetries in the $E$-diagonal basis}

The parameters of the scalar potential given by eq. (2.2) are described in the K space formalism by eqs. (2.11) and (2.12). In particular, under $\mathrm{U}(2)$ basis transformations of eq. (2.5) $\boldsymbol{\xi}$ and $\boldsymbol{\eta}$ transform as real three-vectors and $E$ transforms as a real Cartesian second-rank symmetric tensor under $\mathrm{SO}(3)$, which is related to the $\mathrm{U}(2)$ basis transformation of the scalar fields via eq. (2.9). Starting in a generic basis, one can always transform to a special basis in which $E$ is diagonal,

$$
R_{D} E R_{D}^{\mathrm{T}} \equiv E_{D}=\operatorname{diag}\left(\mu_{1}, \mu_{2}, \mu_{3}\right)
$$

17 Note that in [4] the derivation of the symmetry classes assumes strong stability of the potential, that is, the quartic terms alone guarantee stability. Here we consider the general case including weak and marginal stability where the stability of the potential is guaranteed only after inclusion of the quadratic terms. In fact, the classification of Table Iis valid even for unstable potentials. 


\begin{tabular}{lcl}
\hline rotation matrices in the $E$-diagonal basis & symmetry class & constraints on scalar potential parameters \\
\hline$R(\hat{\boldsymbol{e}}, \pi)$ & $\mathbb{Z}_{2}$ & $\boldsymbol{\xi} \times \hat{\boldsymbol{e}}=\boldsymbol{\eta} \times \hat{\boldsymbol{e}}=0$; eigenvalues of $E$ are unconstrained \\
$R(\hat{\boldsymbol{n}}, \pi), \hat{\boldsymbol{n}} \neq \hat{\boldsymbol{e}}$ & $\mathbb{Z}_{2}$ & $\boldsymbol{\xi} \times \hat{\boldsymbol{n}}=\boldsymbol{\eta} \times \hat{\boldsymbol{n}}=0$; eigenvalues of $E$ are degenerate \\
$R(\hat{\boldsymbol{e}}, \theta), 0<\theta<\pi$ & $\mathrm{U}(1)$ & $\boldsymbol{\xi} \times \hat{\boldsymbol{e}}=\boldsymbol{\eta} \times \hat{\boldsymbol{e}}=0$; eigenvalues of $E$ are doubly-degenerate \\
$R(\hat{\boldsymbol{n}}, \theta), \hat{\boldsymbol{n}} \neq \hat{\boldsymbol{e}}, 0<\theta<\pi$, & $\mathrm{U}(1)$ & $\boldsymbol{\xi} \times \hat{\boldsymbol{n}}=\boldsymbol{\eta} \times \hat{\boldsymbol{n}}=0 ; E=\mu \mathbb{1}_{3}$ \\
$-R(\hat{\boldsymbol{e}}, \pi)$ & $\mathrm{CP} 1$ & $\boldsymbol{\xi} \cdot \hat{\boldsymbol{e}}=\boldsymbol{\eta} \cdot \hat{\boldsymbol{e}}=0 ;$ eigenvalues of $E$ are unconstrained \\
$-R(\hat{\boldsymbol{n}}, \pi), \hat{\boldsymbol{n}} \neq \hat{\boldsymbol{e}}$ & $\mathrm{CP} 1$ & $\boldsymbol{\xi} \cdot \hat{\boldsymbol{n}}=\boldsymbol{\eta} \cdot \hat{\boldsymbol{n}}=0$; eigenvalues of $E$ are degenerate \\
$-R(\hat{\boldsymbol{n}}, 0) \equiv-\mathbb{1}_{3}$, & $\mathrm{CP} 2$ & $\boldsymbol{\xi}=\boldsymbol{\eta}=0 ;$ eigenvalues of $E$ are unconstrained \\
$-R(\hat{\boldsymbol{e}}, \theta), 0<\theta<\pi$ & $\mathrm{CP} 3$ & $\boldsymbol{\xi}=\boldsymbol{\eta}=0 ;$ eigenvalues of $E$ are doubly-degenerate \\
$-R(\hat{\boldsymbol{n}}, \theta), \hat{\boldsymbol{n}} \neq \hat{\boldsymbol{e}}, 0<\theta<\pi$ & $\mathrm{CP} 3[\mathrm{SO}(3)]$ & $\boldsymbol{\xi}=\boldsymbol{\eta}=0 ; E=\mu \mathbb{1}_{3}$ \\
\hline
\end{tabular}

TABLE III: The proper [and improper] rotation matrices $R$ [and $\bar{R}$ ] that generate the symmetry classes in $K$ space, where the scalar fields are defined in the $E$-diagonal basis. The unit vector $\hat{\boldsymbol{e}}$ is any one of the three eigenvectors $\left\{\hat{\boldsymbol{e}}_{1}, \hat{\boldsymbol{e}}_{2}, \hat{\boldsymbol{e}}_{3}\right\}$ of $E$. In the cases of the $\mathbb{Z}_{2}$ and CP1 symmetry classes where $\hat{\boldsymbol{n}} \neq \hat{\boldsymbol{e}}_{i}$ (for $i=1,2,3$ ), $E$ possesses doubly-degenerate eigenvalues if $\hat{\boldsymbol{n}}$ can be expressed as a linear combination of two of the eigenvectors of $E$; otherwise $E=\mu \mathbb{1}_{3}$. The last line of the table corresponds to a special case of the CP3 symmetry class in which all three eigenvalues of $E$ are degenerate. The resulting scalar potential parameter constraints yield an $\mathrm{SO}(3)$-invariant scalar potential.

where the $\mu_{i}$ are the eigenvalues of $E$ and $R_{D} \in \mathrm{SO}(3)$. We begin by assuming that the scalar potential parameters are generic. In this case, the eigenvalues of $E$ are non-degenerate, and $R_{D}$ is unique up to the ordering of its rows and/or columns, which corresponds to a reordering of the diagonal elements of $E_{D}$. The corresponding normalized eigenvectors $E$ are unique (up to an irrelevant multiplicative phase) and will be denoted $\hat{\boldsymbol{e}}_{1}, \hat{\boldsymbol{e}}_{2}$ and $\hat{\boldsymbol{e}}_{3}$. Working in the basis where $E$ is diagonal (henceforth called the $E$-diagonal basis) is equivalent to expressing the matrix $E$ with respect to the orthonormal basis $\left\{\hat{\boldsymbol{e}}_{1}, \hat{\boldsymbol{e}}_{2}, \hat{\boldsymbol{e}}_{3}\right\}$.

Having chosen the $E$-diagonal basis, we now investigate the form of the various THDM symmetry classes. In each case, the symmetry transformation is of the form:

$$
\begin{aligned}
& \mathbf{K}(x) \rightarrow R \mathbf{K}(x), \quad \text { for Higgs family symmetries }, \\
& \mathbf{K}(x) \rightarrow \bar{R} \mathbf{K}\left(x^{\prime}\right), \quad \text { for GCP symmetries },
\end{aligned}
$$

where $R$ is a proper rotation and $\bar{R}$ is an improper rotation. In Table III, we summarize the possible forms for $R$ and $\bar{R}$ and identify the relevant symmetry class. We also indicate the corresponding constraints on the scalar potential parameters. In some cases, the imposition of the symmetry will require that the eigenvalues of $E$ exhibit some degeneracy. If the symmetry imposes no such constraint, we say that the eigenvalues of $E$ are unconstrained (i.e. non-degenerate for generic choices of the parameters of the scalar potential).

We now discuss some of the salient points of Table III. First, the constraints on $\boldsymbol{\xi}$ and $\boldsymbol{\eta}$ are precisely the same as the ones given in Table II. These constraints are determined simply by identifying the eigenvector of $R$ or $\bar{R}$ (if it exists) corresponding to the eigenvalue +1 . Indeed, when no eigenvalue +1 exists (as in the cases of CP2 and CP3), it follows that $\boldsymbol{\xi}=\boldsymbol{\eta}=0$. Second, in the cases of proper and improper rotation matrices parameterized in terms of $\hat{\boldsymbol{n}}=\hat{\boldsymbol{e}}$, where $\hat{\boldsymbol{e}}$ is one of the eigenvectors of $E$, no constraints on the eigenvectors of $E$ arise for the $\mathbb{Z}_{2}$, CP1 and CP2 symmetry classes (in which case $\hat{\boldsymbol{e}}$ can be any one of the three eigenvectors of $E$ ). For the U(1) and CP3 symmetry classes, the eigenvalues of $R(\hat{\boldsymbol{n}}, \theta)$ and $\bar{R} \equiv-R(\hat{\boldsymbol{n}}, \theta)$ are non-degenerate, and the corresponding eigenvectors orthogonal to $\hat{\boldsymbol{n}}$ are complex conjugates of each other. Since $E$ commutes with $R$, the eigenvectors of $R$ are also eigenvectors of $E$. Finally, as the eigenvalues of the real symmetric matrix $E$ must be real, it follows that the eigenvalue of $E$ corresponding to the complex conjugate pair of eigenvectors is doubly-degenerate.

However, if $\hat{\boldsymbol{n}} \neq \hat{\boldsymbol{e}}_{i}$ (for $i=1,2,3$ ), then additional constraints on the eigenvalues of $E$ are imposed. Repeating the arguments of Section 5.1, we see that $\hat{\boldsymbol{n}}$ must also be an eigenvector of $E$. This can only be consistent with $\hat{\boldsymbol{n}} \neq \hat{\boldsymbol{e}}_{i}$ if some of the eigenvalues of $E$ are degenerate, in which case linear combinations of the $\hat{\boldsymbol{e}}_{i}$ within the degenerate subspace are also eigenvectors of $E$. For example, in the case of the $\mathbb{Z}_{2}$ or the CP1 symmetry classes, if $\hat{\boldsymbol{n}}$ is a linear combination of two of the eigenvectors of $E$, then eq. (5.16) yields a two-fold degeneracy in the eigenvalues 
of $E$. Likewise, if $\hat{\boldsymbol{n}}$ is a linear combination of all three of the originally chosen eigenvectors of $E$, then eq. (5.16) yields a three-fold degeneracy in the eigenvalues of $E$, which means that $E$ is proportional to the identity matrix (i.e. $E=\mu \mathbb{1}_{3}$ ). Likewise, in the case of the U(1) or the CP3 symmetry classes, if $\hat{\boldsymbol{n}} \neq \hat{\boldsymbol{e}}_{i}$ (for $i=1,2,3$ ), then the eigenvectors of $R$ or $\bar{R}$, denoted by $\hat{\boldsymbol{m}}$ and $\hat{\boldsymbol{m}}^{*}$ in Section 5.1, are linear combinations of all three of the eigenvectors of $E$ [cf. eq. (A.13)]. Consequently, eq. (5.31) requires that all three eigenvalues of $E$ must be degenerate, i.e. $E=\mu \mathbb{1}_{3}$. Here is a simple example to illustrate the last point. Suppose we work in a coordinate system in which the eigenvectors of $E$ are $\hat{\boldsymbol{e}}_{1}=(1,0,0), \hat{\boldsymbol{e}}_{2}=(0,1,0)$, and $\hat{\boldsymbol{e}}_{3}=(0,0,1)$. One possible choice for the axis of the rotation matrix $R(\hat{\boldsymbol{n}}, \theta)$ is $\hat{\boldsymbol{n}}=\frac{1}{\sqrt{2}}\left(\hat{\boldsymbol{e}}_{1}+\hat{\boldsymbol{e}}_{2}\right)=\frac{1}{\sqrt{2}}(1,1,0)$. In this case, we use eq. A.13) to obtain

$$
\hat{\boldsymbol{m}}=\frac{1}{2}\left(\frac{1}{\sqrt{2}}(1+i),-\frac{1}{\sqrt{2}}(1+i),-1+i\right),
$$

where $\hat{\boldsymbol{m}}$ is the eigenvector of $R(\hat{\boldsymbol{n}}, \theta)$ corresponding to the non-degenerate eigenvalue $e^{i \theta}$ of $R(\hat{\boldsymbol{n}}, \theta)$ [under the assumption that $0<\theta<\pi]$. When we impose the symmetry constraint, $E R(\hat{\boldsymbol{n}}, \theta)=R E(\hat{\boldsymbol{n}}, \theta)$, it follows that $\hat{\boldsymbol{m}}$ must also be an eigenvector of $E$. This is only possible if $E=\mu \mathbb{1}_{3}$, as indicated in the last row of Table III. Any other choice of $\hat{\boldsymbol{n}} \neq \hat{\boldsymbol{e}}_{i}$ (for $i=1,2,3$ ) would lead to the same conclusion.

To summarize, we have examined possible symmetry transformations in the $E$-diagonal basis, in which the vectors $\boldsymbol{\xi}$ and $\boldsymbol{\eta}$ and the matrix $E$ are given with respect to the orthonormal basis constructed from the eigenvectors of $E$, namely $\left\{\hat{\boldsymbol{e}}_{1}, \hat{\boldsymbol{e}}_{2}, \hat{\boldsymbol{e}}_{3}\right\}$. Applying a symmetry transformation $\pm R(\hat{\boldsymbol{n}}, \theta)$, where $\hat{\boldsymbol{n}} \neq \hat{\boldsymbol{e}}_{i}$ (for $i=1,2,3$ ), yields the symmetry classes $\mathbb{Z}_{2}, \mathrm{U}(1), \mathrm{CP} 1, \mathrm{CP} 2$ and CP3, but with extra degeneracies among the eigenvalues of $E$. Nevertheless, these extra degeneracies do not correspond in general to new enhanced symmetry classes as argued in Section 5.2. In particular, symmetry transformations defined with respect to a specific basis choice can yield constraints on the scalar potential parameters that are not stable with respect to renormalization group running.

Of course, one can always redefine the scalar fields of the tree-level scalar potential to achieve a particular choice of basis. However, once higher order corrections are taken into account, the energy scale of the scalar potential parameters becomes relevant. For example, the diagonalization required to achieve the $E$-diagonal basis depends on the renormalization scale $M$. In general, the eigenvalues and the directions of the eigenvectors of $E$ will not remain fixed under a change in the renormalization scale. Thus, parameter constraints defined in some specific basis relative to some scale $M$ will not automatically be preserved at some other scale $M^{\prime} \neq M$. When discussing restrictions on parameters of the tree-level scalar potential due to possible symmetries, one has to check explicitly that they are preserved by renormalization group evolution.

As an example, we consider the GCP transformation

$$
\left(\begin{array}{l}
\varphi_{1}(x) \\
\varphi_{2}(x)
\end{array}\right) \rightarrow\left(\begin{array}{cc}
e^{-i \omega} & 0 \\
0 & e^{i \omega}
\end{array}\right)\left(\begin{array}{l}
\varphi_{1}^{*}\left(x^{\prime}\right) \\
\varphi_{2}^{*}\left(x^{\prime}\right)
\end{array}\right)
$$

for some fixed $\omega$ with $0<\omega<\pi / 2$, which corresponds to an improper rotation in $K$ space given by

$$
\bar{R}_{\omega}=\left(\begin{array}{ccc}
\cos 2 \omega & \sin 2 \omega & 0 \\
\sin 2 \omega & -\cos 2 \omega & 0 \\
0 & 0 & 1
\end{array}\right) .
$$

We require this to be a symmetry. Using eqs. (A.8), A.10) and A.11, it follows that

$$
\bar{R}_{\omega} \equiv-R(\hat{\boldsymbol{n}}, \pi), \quad \text { with } \hat{\boldsymbol{n}}=(\sin \omega,-\cos \omega, 0),
$$

which means that $\bar{R}_{\omega}$ generates a CP1 transformation. If we apply this transformation in the $E$-diagonal basis, where $\hat{\boldsymbol{e}}_{1}=(1,0,0), \hat{\boldsymbol{e}}_{2}=(0,1,0)$, and $\hat{\boldsymbol{e}}_{3}=(0,0,1)$, then $\hat{\boldsymbol{n}}=\sin \omega \hat{\boldsymbol{e}}_{1}-\cos \omega \hat{\boldsymbol{e}}_{2}$ must also be an eigenvector of $E$. Hence in the $E$-diagonal basis, $E=\operatorname{diag}\left(\mu_{1}, \mu_{1}, \mu_{3}\right)$ possesses doubly-degenerate eigenvalues.

However, the existence of doubly-degenerate eigenvalues is not stable under renormalization group evolution. To verify this statement, we shall work in a real basis (i.e. a basis in which all scalar potential parameters exhibited 
in eq. (2.2) are real), which is stable under renormalization group running. Using eq. (2.12), the matrix $E$ then takes the form:

$$
E=\frac{1}{4}\left(\begin{array}{ccc}
\lambda_{4}+\lambda_{5} & 0 & \lambda_{6}-\lambda_{7} \\
0 & \lambda_{4}-\lambda_{5} & 0 \\
\lambda_{6}-\lambda_{7} & 0 & \frac{1}{2}\left(\lambda_{1}+\lambda_{2}\right)-\lambda_{3}
\end{array}\right)
$$

where $\lambda_{5}, \lambda_{6}$ and $\lambda_{7}$ are real. In the $E$-diagonal basis, $\lambda_{6}=\lambda_{7}$. Applying the symmetry constraints, eq. (3.40), imposed by $\bar{R}_{\omega}$, yields $\lambda_{5}=0$ and we see that $E$ possesses a doubly-degenerate eigenvalue, $\lambda_{4}$. We first note that the diagonal form for $E$ is not preserved under renormalization group running. In particular, in the real basis,

$$
8 \pi^{2} \beta_{\lambda_{6}-\lambda_{7}}=\left(\lambda_{6}-\lambda_{7}\right)\left[3\left(\lambda_{1}+\lambda_{2}\right)+2 \lambda_{4}+4 \lambda_{5}\right]+3\left(\lambda_{6}+\lambda_{7}\right)\left(\lambda_{1}-\lambda_{2}\right) .
$$

Since $\lambda_{1} \neq \lambda_{2}$ in general, we see that the diagonal form for $E$ is not stable. However, to check whether the CP1 symmetry is enhanced, one must examine the eigenvalues of eq. (5.66) to see whether the doubly-degenerate eigenvalue is preserved or not under the renormalization group running. A straightforward computation shows that eq. (5.66) has a doubly-degenerate eigenvalue if

$$
D \equiv \lambda_{5}\left(\lambda_{1}+\lambda_{2}-2 \lambda_{3}-2 \lambda_{4}+2 \lambda_{5}\right)-\left(\lambda_{6}-\lambda_{7}\right)^{2}=0 .
$$

The beta function for $D$ is given by

$$
\begin{aligned}
\beta_{D} & =\left(\lambda_{1}+\lambda_{2}-2 \lambda_{3}-2 \lambda_{4}+2 \lambda_{5}\right) \beta_{\lambda_{5}}+\lambda_{5} \beta_{\lambda_{1}+\lambda_{2}-2 \lambda_{3}-2 \lambda_{4}+2 \lambda_{5}}-2\left(\lambda_{6}-\lambda_{7}\right) \beta_{\lambda_{6}-\lambda_{7}} \\
& =\lambda_{5}\left(\beta_{\lambda_{1}}+\beta_{\lambda_{2}}-2 \beta_{\lambda_{3}}-2 \beta_{\lambda_{4}}\right)+\left(\lambda_{1}+\lambda_{2}-2 \lambda_{3}-2 \lambda_{4}+4 \lambda_{5}\right) \beta_{\lambda_{5}}-2\left(\lambda_{6}-\lambda_{7}\right)\left(\beta_{\lambda_{6}}-\beta_{\lambda_{7}}\right) .
\end{aligned}
$$

Inserting the corresponding RGEs in the real basis [18, 48, 66, 67] yields:

$8 \pi^{2} \beta_{D}=4 D\left(\lambda_{1}+\lambda_{2}+\lambda_{3}+2 \lambda_{4}-\lambda_{5}\right)+3\left(\lambda_{6}+\lambda_{7}\right)^{2}\left(\lambda_{1}+\lambda_{2}-2 \lambda_{3}-2 \lambda_{4}+2 \lambda_{5}\right)+3\left(\lambda_{1}-\lambda_{2}\right)\left[\lambda_{5}\left(\lambda_{1}-\lambda_{2}\right)-2 \lambda_{6}^{2}+2 \lambda_{7}^{2}\right]$.

Indeed, as long as $\lambda_{1}=\lambda_{2}$ and $\lambda_{7}=-\lambda_{6}$ is not satisfied, we see that $D=0$ is not a fixed point of eq. (5.70). We recognize $\lambda_{1}=\lambda_{2}$ and $\lambda_{7}=-\lambda_{6}$ as the constraints on the dimensionless parameters of the scalar potential in the exceptional region of the parameter space (ERPS) identified in 50]. When we impose these ERPS conditions, the CP1 symmetry is promoted to a CP3 symmetry ${ }^{18}$ (modulo possible soft-symmetry breaking squared-mass terms). Outside of the ERPS, the double-degeneracy of the eigenvalue of $E$ is not a renormalization group invariant, and the CP1 symmetry is not enhanced.

In Table III, when $\hat{\boldsymbol{n}} \neq \hat{\boldsymbol{e}}_{i}$ (for $i=1,2,3$ ), the enhanced degeneracies in the eigenvalues of $E$ are not stable with respect to renormalization group running in the cases of the $\mathbb{Z}_{2}, \mathrm{U}(1)$ and $\mathrm{CP} 1$ symmetry classes. However, in the case of the CP3 symmetry class, the constraints on the scalar potential parameters coincide with eq. (5.52), which are the same constraints imposed by the $\mathrm{SO}(3)$-invariant scalar potential. In this case, the resulting parameter constraints are invariant with respect to renormalization group running. That is, the CP3 symmetry class with an enhanced degeneracy of the eigenvalues of $E$ is promoted to the $S O(3)$ symmetry class. In particular, it is possible to generate an $\mathrm{SO}(3)$ Higgs family symmetry by applying any particular CP3 transformation with $\hat{\boldsymbol{n}} \neq \hat{\boldsymbol{e}}_{i}$ (for $i=1,2,3$ ) in the $E$-diagonal basis. This seems to be in conflict with the results of Section 5.1, where it was shown that two non-collinear rotation matrices are necessary in order to generate the $\mathrm{SO}(3)$ symmetry class in a generic basis. However, given that an independent rotation matrix $R_{D}$ is required [cf. eq. (5.59)] in order to define the $E$-diagonal basis, it is perhaps not surprising that the $\mathrm{SO}(3)$ symmetry class can be generated by applying a single (improper) rotation matrix in the $E$-diagonal basis.

${ }^{18}$ In the E-diagonal basis, the ERPS conditions on the dimensionless couplings of the scalar potential corresponds to $\lambda_{1}=\lambda_{2}$ and $\lambda_{5}=\lambda_{6}=\lambda_{7}=0$. One is free to make a change of basis that simultaneously interchanges the rows and columns of $E$ while keeping $E$ diagonal. In the new basis, $\lambda_{1}=\lambda_{2}$ and $\lambda_{6}=\lambda_{7}=0$ are maintained, while $\lambda_{5}=0$ is transformed to $\lambda_{5}=\lambda_{1}-\lambda_{3}-\lambda_{4}$. The latter reproduces the conditions for a CP3-symmetric scalar potential given in Table I of [50]. 
The classification of the possible THDM symmetries is best done in a generic basis, where the structure of the various symmetry classes is transparent, and the resulting constraints on the scalar potential parameters can be obtained that are covariant with respect to basis transformations. Although it is possible to perform an analysis of symmetry classes in a specific basis (the $E$-diagonal basis is a convenient choice to consider for this purpose), the resulting classification is complicated by enhanced symmetry constraints that are typically not renormalization group invariant. Such enhanced symmetry points in the scalar potential parameter space are accidental in nature and are not indicative of any new symmetry structures.

\section{CONCLUSIONS}

It is known that there are only six classes of symmetry-constrained potentials in the THDM [44]. Specific implementations were later explored in [50], using Higgs-family and generalized CP (GCP) symmetries of the THDM potential. In this paper, we have presented an analysis of the symmetry classes, which applies to completely general scalar potentials. That is, our analysis applies to scalar potentials that are stable in the strong, weak, or marginal sense, or even unstable. Furthermore, we have pursued the geometric $K$ space interpretation of Higgs-family and GCP transformations [38, 40]; the former are proper $\mathrm{SO}(3)$ rotations and the latter are improper rotations of the field bilinears in $K$ space. We have constructed the relevant rotations and shown explicitly their effects on the Higgs scalar potential. This combines some known results with new ones into a unified scheme and sets the framework for our analysis.

The following results have thus been obtained. We have clarified the relation of the classifications of GCP transformations of [45] and [50]. We have given a simple geometric proof relating $\mathrm{SO}(3)$ rotations to two reflections through planes and improper rotations to one or three reflections through planes in $K$ space. We have shown that any Higgsfamily transformation can be considered as a product of two CP1 transformations and any GCP transformation is either a CP1 transformation or a product of three CP1 transformations. Based on this result we have provided a geometric interpretation of the surprising result presented in [50] that all Higgs-family and GCP symmetries in the THDM can be generated from suitable CP1 symmetries.

\section{Acknowledgments}

The work of P.M.F. is supported in part by the Portuguese Fundação para a Ciência e a Tecnologia (FCT) under contract PTDC/FIS/70156/2006. The work of H.E.H. is supported in part by the U.S. Department of Energy, under grant number DE-FG02-04ER41268 and in part by a Humboldt Research Award sponsored by the Alexander von Humboldt Foundation. The work of J.P.S. is funded by the Portuguese FCT through the projects CERN/FP/109305/2009 and U777-Plurianual, and by the EU RTN project Marie Curie: MRTN-CT-2006-035505. The work of M.M. is supported by Deutsche Forschungsgemeinschaft project number NA296/5-1. H.E.H. is grateful for the hospitality of the Bethe Center for Theoretical Physics at the Physikalisches Institut der Universität Bonn, where part of this work was completed. 


\section{Appendix A: Properties of $3 \times 3$ proper rotation matrices}

In this Appendix, we review the properties of $3 \times 3$ proper rotation matrices. Most of this material is standard and can be found in $[68-70]$.

The most general three-dimensional proper rotation is represented by an $\mathrm{SO}(3)$ matrix, $R(\hat{\boldsymbol{n}}, \theta)$, whose form is uniquely specified by an axis of rotation, $\hat{\boldsymbol{n}}$, and a rotation angle $\theta$. Conventionally, a positive rotation angle corresponds to a counterclockwise rotation. The direction of the axis is determined by the right hand rule. Simple geometrical considerations imply that:

$$
\begin{gathered}
R(\hat{\boldsymbol{n}}, \theta+2 \pi k)=R(\hat{\boldsymbol{n}}, \theta), \quad k=0, \pm 1 \pm 2 \ldots, \\
{[R(\hat{\boldsymbol{n}}, \theta)]^{-1}=R(\hat{\boldsymbol{n}},-\theta)=R(-\hat{\boldsymbol{n}}, \theta) .}
\end{gathered}
$$

Combining these two results, it follows that

$$
R(\hat{\boldsymbol{n}}, 2 \pi-\theta)=R(-\hat{\boldsymbol{n}}, \theta),
$$

which implies that any three-dimensional rotation can be described by a counterclockwise rotation by $\theta$ about an arbitrary axis $\hat{\boldsymbol{n}}$, where $0 \leq \theta \leq \pi \cdot{ }^{19}$ However, for $\theta=\pi$ eq. (A.3) yields:

$$
R(\hat{\boldsymbol{n}}, \pi)=R(-\hat{\boldsymbol{n}}, \pi)
$$

which means that for the special case of $\theta=\pi, R(\hat{\boldsymbol{n}}, \pi)$ and $R(-\hat{\boldsymbol{n}}, \pi)$ represent the same rotation. Finally, if $\theta=0$, then $R(\hat{\boldsymbol{n}}, 0)=\mathbb{1}_{3}$ is the identity operator, independently of the direction of $\hat{\boldsymbol{n}}$.

An explicit form for a general three-dimensional proper rotation is given by:

$$
R_{i j}(\hat{\boldsymbol{n}}, \theta)=\delta_{i j} \cos \theta+n_{i} n_{j}(1-\cos \theta)-\epsilon_{i j k} n_{k} \sin \theta .
$$

This result simplifies for the case of $\theta=\pi$,

$$
R_{i j}(\hat{\boldsymbol{n}}, \pi)=2 n_{i} n_{j}-\delta_{i j}
$$

It is noteworthy that $R(\hat{\boldsymbol{n}}, \theta)$ is a symmetric matrix if and only if $\theta=0(\bmod \pi)$.

Given the $\mathrm{SO}(3)$ matrix $R(\hat{\boldsymbol{n}}, \theta)$, one can determine the corresponding angle of rotation $\theta$ and axis of rotation $\hat{\boldsymbol{n}}$. By taking the trace of eq. (A.5), we immediately obtain

$$
\operatorname{Tr} R(\hat{\boldsymbol{n}}, \theta)=1+2 \cos \theta=1+e^{i \theta}+e^{-i \theta} .
$$

It immediately follows that

$$
\cos \theta=\frac{1}{2}(\operatorname{Tr} R-1),
$$

which determines $\theta$ uniquely in the convention that $0 \leq \theta \leq \pi$. The axis of rotation is given by:

$$
\hat{\boldsymbol{n}}=\frac{1}{\sqrt{(3-\operatorname{Tr} R)(1+\operatorname{Tr} R)}}\left(R_{32}-R_{23}, R_{13}-R_{31}, R_{21}-R_{12}\right), \quad \text { for } \theta \neq 0 \bmod \pi .
$$

For $\theta=0(\bmod \pi), R(\hat{\boldsymbol{n}}, \theta)$ is symmetric and cannot be determined from eq. (A.9). In this case, eq. (A.8) determines whether $\cos \theta=+1$ or $\cos \theta=-1$. If $\cos \theta=+1$, then $R_{i j}=\delta_{i j}$ and the axis $\hat{\boldsymbol{n}}$ is undefined. If $\cos \theta=-1$, then eq. A.6) determines the direction of $\hat{\boldsymbol{n}}$ up to an overall sign. That is,

$\hat{\boldsymbol{n}}$ is undetermined if $\theta=0$,

$$
\hat{\boldsymbol{n}}=\left(\epsilon_{1} \sqrt{\frac{1}{2}\left(1+R_{11}\right)}, \epsilon_{2} \sqrt{\frac{1}{2}\left(1+R_{22}\right)}, \epsilon_{3} \sqrt{\frac{1}{2}\left(1+R_{33}\right)}\right), \quad \text { if } \theta=\pi
$$

19 In the convention adopted here, the overall sign of $\hat{\boldsymbol{n}}$ is meaningful for $\theta \neq 0 \bmod \pi$. 
where the individual signs $\epsilon_{i}= \pm 1$ are determined up to an overall sign via ${ }^{20}$

$$
\epsilon_{i} \epsilon_{j}=\frac{R_{i j}}{\sqrt{\left(1+R_{i i}\right)\left(1+R_{j j}\right)}}, \quad \text { for fixed } i \neq j, R_{i i} \neq-1, R_{j j} \neq-1 .
$$

The ambiguity of the overall sign of $\hat{\boldsymbol{n}}$ sign is not significant, since $R(\hat{\boldsymbol{n}}, \pi)$ and $R(-\hat{\boldsymbol{n}}, \pi)$ represent the same rotation as noted above [cf. eq. A.4 ].

Since the axis of rotation $\hat{\boldsymbol{n}}$ is invariant under all rotations $R(\hat{\boldsymbol{n}}, \theta)$, it follows that $\hat{\boldsymbol{n}}$ is an eigenvector of $R(\hat{\boldsymbol{n}}, \theta)$ with corresponding eigenvalue +1 ,

$$
R(\hat{\boldsymbol{n}}, \theta) \hat{\boldsymbol{n}}=\hat{\boldsymbol{n}} .
$$

Moreover, since $R(\hat{\boldsymbol{n}}, \theta)$ is also a unitary matrix, we know that its eigenvalues are pure phases. Combining eq. (A.7) with $\operatorname{det} R(\hat{\boldsymbol{n}}, \theta)=1$, it follows that the individual eigenvalues of $R(\hat{\boldsymbol{n}}, \theta)$ must be $1, e^{i \theta}$ and $e^{-i \theta}$. For $\theta \neq 0(\bmod \pi)$, these three eigenvalues are non-degenerate. In this case, it is convenient to introduce normalized eigenvectors $\hat{\boldsymbol{m}}$ and $\hat{\boldsymbol{m}}^{*}$, corresponding to the eigenvalues $e^{i \theta}$ and $e^{-i \theta}$, respectively. Note that whereas $\hat{\boldsymbol{n}}$ is a real vector corresponding to the axis of rotation, $\hat{\boldsymbol{m}}$ and its complex conjugate are complex vectors. The vectors $\hat{\boldsymbol{n}}, \hat{\boldsymbol{m}}$ and $\hat{\boldsymbol{m}}^{*}$ are mutually orthonormal with respect to the inner product of a complex vector space (e.g., $\hat{\boldsymbol{n}} \cdot \hat{\boldsymbol{m}}=\hat{\boldsymbol{m}} \cdot \hat{\boldsymbol{m}}=0$ and $\hat{\boldsymbol{m}} \cdot \hat{\boldsymbol{m}}^{*}=1$ ). Explicitly, we have

$$
\hat{\boldsymbol{m}}=\frac{1}{\sqrt{2}}\left(n_{3}+\frac{i n_{2}\left(n_{1}-i n_{2}\right)}{1+n_{3}},-i n_{3}-\frac{i n_{1}\left(n_{1}-i n_{2}\right)}{1+n_{3}},-\left(n_{1}-i n_{2}\right)\right), \quad \text { for } \hat{\boldsymbol{n}} \neq-\hat{\boldsymbol{z}},
$$

up to an overall phase factor that can be fixed by convention. This form is not very useful as $n_{3} \rightarrow-1$. However, we can use eq. (A.2) to obtain (up to an overall phase)

$$
\hat{\boldsymbol{m}}=\frac{1}{\sqrt{2}}\left(-n_{3}-\frac{i n_{2}\left(n_{1}+i n_{2}\right)}{1-n_{3}},-i n_{3}+\frac{i n_{1}\left(n_{1}+i n_{2}\right)}{1-n_{3}}, n_{1}+i n_{2}\right), \quad \text { for } \hat{\boldsymbol{n}} \neq \hat{\boldsymbol{z}} .
$$

Clearly, the eigenvectors $\hat{\boldsymbol{n}}, \hat{\boldsymbol{m}}$ and $\hat{\boldsymbol{m}}^{*}$ are independent of the value of the rotation angle $\theta$. In numerical work, it is convenient to use eq. (A.13) for $n_{3} \geq 0$ and eq. (A.14) for $n_{3} \leq 0$. One can check that for $\hat{\boldsymbol{n}} \neq \pm \hat{\boldsymbol{z}}$, eqs. (A.13) and (A.14) are identical up to an irrelevant multiplicative overall phase.

In the case of $\theta=\pi, R(\hat{\boldsymbol{n}}, \pi)$ possesses one non-degenerate eigenvalue +1 and two degenerate eigenvalues -1 . The former is associated with the axis of rotation [cf. eq. (A.12)]. The eigenvectors corresponding to the degenerate eigenvalues, denoted below by $\hat{\boldsymbol{m}}_{1}$ and $\hat{\boldsymbol{m}}_{2} \equiv \hat{\boldsymbol{n}} \times \hat{\boldsymbol{m}}_{1}$, can be chosen to be real and orthonormal. A convenient choice is: ${ }^{21}$

$$
\hat{\boldsymbol{m}}_{\mathbf{1}}=\left(n_{3}+\frac{n_{2}^{2}}{1+n_{3}}, \frac{-n_{1} n_{2}}{1+n_{3}},-n_{1}\right), \quad \hat{\boldsymbol{m}}_{2}=\left(\frac{-n_{1} n_{2}}{1+n_{3}}, n_{3}+\frac{n_{1}^{2}}{1+n_{3}},-n_{2}\right) .
$$

However, any other orthonormal pair of vectors constructed from linear combinations of $\hat{\boldsymbol{m}}_{1}$ and $\hat{\boldsymbol{m}}_{2}$ would be equally suitable. Finally, in the case of $\theta=0, R(\hat{\boldsymbol{n}}, \pi)=\mathbb{1}_{3}$ possesses three degenerate eigenvalues +1 .

Finally, we prove an important result that is needed in the text. Let $\widetilde{R} \in \mathrm{SO}(3)$ such that

$$
\hat{\boldsymbol{n}}^{\prime}=\widetilde{R} \hat{\boldsymbol{n}}
$$

Then

$$
R\left(\hat{\boldsymbol{n}}^{\prime}, \theta\right)=\widetilde{R} R(\hat{\boldsymbol{n}}, \theta) \widetilde{R}^{\mathrm{T}}
$$

To prove eq. A.17), we first note that

$$
\operatorname{Tr} R\left(\hat{\boldsymbol{n}}^{\prime}, \theta\right)=\operatorname{Tr}\left[\widetilde{R} R(\hat{\boldsymbol{n}}, \theta) \widetilde{R}^{\mathrm{T}}\right]=\operatorname{Tr} R(\hat{\boldsymbol{n}}, \theta),
$$

${ }^{20}$ If $R_{i i}=-1$, where $i$ is a fixed index, then $n_{i}=0$, in which case the corresponding $\epsilon_{i}$ is not well-defined.
${ }^{21}$ In this case, any problem involving $n_{3}=-1$ can be avoided simply by employing eq. A.4. 
using the cyclicity of the trace and $\widetilde{R} \widetilde{R}^{T}=\mathbb{1}_{3}$. It follows from eq. A.7) that the angle of rotation of $R(\hat{\boldsymbol{n}}, \theta)$ and $\widetilde{R} R(\hat{\boldsymbol{n}}, \theta) \widetilde{R}^{\mathrm{T}}$ must be the same. Next, we use eq. (A.12) to determine the axis of rotation of $\widetilde{R} R(\hat{\boldsymbol{n}}, \theta) \widetilde{R}^{\mathrm{T}}$,

$$
\widetilde{R} R(\hat{\boldsymbol{n}}, \theta) \widetilde{R}^{\mathrm{T}}(\widetilde{R} \hat{\boldsymbol{n}})=\widetilde{R} R(\hat{\boldsymbol{n}}, \theta) \hat{\boldsymbol{n}}=\widetilde{R} \hat{\boldsymbol{n}}
$$

which implies that $\widetilde{R} \hat{\boldsymbol{n}}$ is an eigenvector of $\widetilde{R} R(\hat{\boldsymbol{n}}, \theta) \widetilde{R}^{\mathrm{T}}$ with eigenvalue +1 . If $\theta \neq 0$, then the eigenvalue +1 is non-degenerate, in which case $\widetilde{R} \hat{\boldsymbol{n}}$ is the axis of rotation of $R\left(\hat{\boldsymbol{n}}^{\prime}, \theta\right)$, and eqs. (A.16) and (A.17) are confirmed. If $\theta=0$, then eq. (A.17) is trivially satisfied.

Specializing to the case of $\hat{\boldsymbol{n}}=\hat{\boldsymbol{z}}$, it then follows that:

$$
R(\hat{\boldsymbol{n}}, \theta)=\widetilde{R} R(\hat{z}, \theta) \widetilde{R}^{T}, \quad \text { where } \hat{\boldsymbol{n}} \equiv \widetilde{R} \hat{\boldsymbol{z}} .
$$

[1] K. Nakamura et al. [Particle Data Group], "Review of particle physics," J. Phys. G 37, 075021 (2010).

[2] J.F. Gunion, H.E. Haber, G.L. Kane and S. Dawson, The Higgs Hunter's Guide (Westview Press, Boulder, CO, 2000).

[3] T.D. Lee, "A theory of spontaneous T violation," Phys. Rev. D 8, 1226 (1973).

[4] T.D. Lee, "CP nonconservation and spontaneous symmetry breaking," Phys. Rept. 9, 143 (1974).

[5] R.D. Peccei and H.R. Quinn, "CP conservation in the presence of instantons," Phys. Rev. Lett. 38, 1440 (1977).

[6] R.D. Peccei and H.R. Quinn, "Constraints imposed by CP conservation in the presence of instantons," Phys. Rev. D 16, 1791 (1977).

[7] P. Fayet, "A gauge theory of weak and electromagnetic interactions with spontaneous parity breaking," Nucl. Phys. B 78, 14 (1974);

[8] P. Fayet, "Supergauge invariant extension of the Higgs mechanism and a model for the electron and its neutrino," Nucl. Phys. B 90, 104 (1975).

[9] K. Inoue, A. Kakuto, H. Komatsu and S. Takeshita, "Aspects of grand unified models with softly broken supersymmetry," Prog. Theor. Phys. 68, 927 (1982) [Erratum-ibid. 70, 330 (1983)]; "Renormalization of supersymmetry breaking parameters revisited," Prog. Theor. Phys. 71, 413 (1984).

[10] R.A. Flores and M. Sher, "Higgs masses in the standard, multi-Higgs and supersymmetric Models," Annals Phys. 148, 95 (1983).

[11] J.F. Gunion and H.E. Haber, "Higgs bosons in supersymmetric models. I," Nucl. Phys. B 272, 1 (1986) [Erratum-ibid. B 402, 567 (1993)].

[12] N.G. Deshpande and E. Ma, "Pattern Of Symmetry Breaking With Two Higgs Doublets," Phys. Rev. D 18, 2574 (1978).

[13] H. Georgi, "A Model Of Soft CP Violation," Hadronic J. 1, 155 (1978).

[14] H.E. Haber, G.L. Kane and T. Sterling, "The Fermion Mass Scale And Possible Effects Of Higgs Bosons On Experimental Observables," Nucl. Phys. B 161, 493 (1979).

[15] J.F. Donoghue and L. F. Li, "Properties Of Charged Higgs Bosons," Phys. Rev. D 19, 945 (1979).

[16] E. Golowich and T.C. Yang, "Charged Higgs Bosons And Decays Of Heavy Flavored Mesons," Phys. Lett. B 80, 245 (1979).

[17] L.J. Hall and M.B. Wise, "Flavor Changing Higgs Boson Couplings," Nucl. Phys. B 187, 397 (1981).

[18] H.E. Haber and R. Hempfling, "The Renormalization group improved Higgs sector of the minimal supersymmetric model," Phys. Rev. D 48, 4280 (1993) arXiv:hep-ph/9307201. We alert the reader to one typographical error in Appendix A of this paper. In eq. (A2), replace $12 \lambda_{6}^{2}$ with $12 \lambda_{7}^{2}$. Note that the renormalization equations given in Appendix A were derived for the CP-conserving THDM in which all scalar potential parameters are real.

[19] G. Cvetic, "CP violation in bosonic sector of SM with two Higgs doublets," Phys. Rev. D 48, 5280 (1993) arXiv:hep-ph/9309202.

[20] F.J. Botella and J.P. Silva, "Jarlskog-like invariants for theories with scalars and fermions," Phys. Rev. D 51, (1995) 3870 arXiv:hep-ph/9411288.

[21] L. Lavoura and J.P. Silva, "Fundamental CP violating quantities in a SU(2) x U(1) model with many Higgs doublets," Phys. Rev. D 50, 4619 (1994) arXiv:hep-ph/9404276.

[22] L. Lavoura, "Signatures of discrete symmetries in the scalar sector," Phys. Rev. D 50, 7089 (1994) arXiv:hep-ph/9405307.

[23] W. Bernreuther and O. Nachtmann, "Flavour dynamics with general scalar fields," Eur. Phys. J. C 9, 319 (1999) arXiv:hep-ph/9812259. 


\section{A PROPERTIES OF $\mathbf{3} \times \mathbf{3}$ PROPER ROTATION MATRICES}

[24] I.F. Ginzburg and M. Krawczyk, "Symmetries of two Higgs doublet model and CP violation," Phys. Rev. D 72, 115013 (2005) arXiv:hep-ph/0408011.

[25] S. Davidson and H.E. Haber, "Basis-independent methods for the two-Higgs-doublet model," Phys. Rev. D 72, 035004 (2005) [Erratum-ibid. D 72, 099902 (2005)] arXiv:hep-ph/0504050].

[26] J.F. Gunion and H. E. Haber, "Conditions for CP-violation in the general two-Higgs-doublet model," Phys. Rev. D 72, 095002 (2005) arXiv:hep-ph/0506227.

[27] H.E. Haber and D. O'Neil, "Basis-independent methods for the two-Higgs-doublet model. II: The significance of $\tan \beta$," Phys. Rev. D 74, 015018 (2006) arXiv:hep-ph/0602242.

[28] R. Barbieri and L.J. Hall, "Improved naturalness and the two Higgs doublet model," arXiv:hep-ph/0510243.

[29] G.C. Branco, M.N. Rebelo and J.I. Silva-Marcos, "CP-odd invariants in models with several Higgs doublets," Phys. Lett. B 614, 187 (2005) arXiv:hep-ph/0502118.

[30] A. Barroso, P.M. Ferreira and R. Santos, "Charge and CP symmetry breaking in two Higgs doublet models," Phys. Lett. B 632, 684 (2006) arXiv:hep-ph/0507224.

[31] R. Barbieri, L.J. Hall and V.S. Rychkov, "Improved naturalness with a heavy Higgs: An alternative road to LHC physics," Phys. Rev. D 74, 015007 (2006) arXiv:hep-ph/0603188.

[32] L. Fromme, S.J. Huber and M. Seniuch, "Baryogenesis in the two-Higgs doublet model," JHEP 0611, 038 (2006) arXiv:hep-ph/0605242.

[33] A. Barroso, P.M. Ferreira and R. Santos, "Neutral minima in two-Higgs doublet models," Phys. Lett. B 652, 181 (2007) arXiv:hep-ph/0702098.

[34] J.M. Gerard and M. Herquet, "A twisted custodial symmetry in the two-Higgs-doublet model," Phys. Rev. Lett. 98, 251802 (2007) arXiv:hep-ph/0703051.

[35] F. Mahmoudi and O. Stal, "Flavor constraints on the two-Higgs-doublet model with general Yukawa couplings," arXiv:0907.1791 [hep-ph]].

[36] P.M. Ferreira and J.P. Silva, "A Two-Higgs Doublet Model With Remarkable CP Properties", accepted for publication in Eur. Phys. J. C, arXiv:1001.0574v2 [hep-ph]].

[37] J. Velhinho, R. Santos and A. Barroso, "Tree level vacuum stability in two-Higss doublet models," Phys. Lett. B 322, 213 (1994).

[38] F. Nagel, "New aspects of gauge-boson couplings and the Higgs sector," Ph. D. thesis (University of Heidelberg, 2004), available from http://archiv.ub.uni-heidelberg.de/archiv/4803.

[39] I.P. Ivanov, "Two-Higgs-doublet model from the group-theoretic perspective," Phys. Lett. B632, 360 (2006) hep-ph/0507132.

[40] M. Maniatis, A. von Manteuffel, O. Nachtmann and F. Nagel, "Stability and symmetry breaking in the general two-Higgsdoublet model," Eur. Phys. J. C 48, 805 (2006) arXiv:hep-ph/0605184.

[41] M. Maniatis, A. von Manteuffel and O. Nachtmann, "Determining the global minimum of Higgs potentials via Groebner bases-applied to the NMSSM," Eur. Phys. J. C 49, 1067 (2007) arXiv:hep-ph/0608314].

[42] C.C. Nishi, "CP violation conditions in N-Higgs-doublet potentials," Phys. Rev. D 74, 036003 (2006) [Erratum-ibid. D 76, 119901 (2007)] arXiv:hep-ph/0605153].

[43] I.P. Ivanov, "Minkowski space structure of the Higgs potential in 2HDM," Phys. Rev. D 75, 035001 (2007) [Erratum-ibid. D 76, 039902 (2007)] arXiv:hep-ph/0609018].

[44] I.P. Ivanov, "Minkowski space structure of the Higgs potential in 2HDM. II. Minima, symmetries, and topology," Phys. Rev. D77, 015017 (2008) arXiv:0710.3490 [hep-ph]].

[45] M. Maniatis, A. von Manteuffel and O. Nachtmann, "CP Violation in the general two-Higgs-doublet Model: a geometric view," Eur. Phys. J. C 57, 719 (2008) arXiv:0707.3344 [hep-ph]].

[46] M. Maniatis, A. von Manteuffel and O. Nachtmann, "A new type of CP symmetry, family replication and fermion mass hierarchies," Eur. Phys. J. C 57, 739 (2008) arXiv:0711.3760 [hep-ph]].

[47] M. Maniatis and O. Nachtmann, "On the phenomenology of a two-Higgs-doublet model with maximal CP symmetry at the LHC," JHEP 0905, 028 (2009) arXiv:0901.4341 [hep-ph]].

[48] E. Ma and M. Maniatis, "Symbiotic Symmetries of the Two-Higgs-Doublet Model," Phys. Lett. B 683, 33 (2010) arXiv:0909.2855 [hep-ph]].

[49] M. Maniatis and O. Nachtmann, "On the phenomenology of a two-Higgs-doublet model with maximal CP symmetry at the LHC, II: radiative effects," arXiv:0912.2727 [hep-ph]].

[50] P.M. Ferreira, H.E. Haber and J.P. Silva, "Generalized CP symmetries and special regions of parameter space in the two-Higgs-doublet model," Phys. Rev. D 79, 116004 (2009) [arXiv:0902.1537 [hep-ph]]. 
[51] G. Ecker, W. Grimus and W. Konetschny, "Quark mass matrices in left-right symmetric gauge theories," Nucl. Phys. B 191, 465 (1981).

[52] G. Ecker, W. Grimus and H. Neufeld, "Spontaneous CP violation in left-right symmetric gauge theories," Nucl. Phys. B 247, 70 (1984).

[53] G. Ecker, W. Grimus and H. Neufeld, "A standard form for generalized CP transformations," J. Phys. A 20, L807 (1987).

[54] H. Neufeld, W. Grimus and G. Ecker, "Generalized CP invariance, neutral flavor conservation and the structure of the mixing matrix," Int. J. Mod. Phys. A 3, 603 (1988).

[55] G.C. Branco, L. Lavoura and J.P. Silva, CP Violation (Oxford University Press, Oxford, UK, 1999).

[56] O. Nachtmann, Elementary Particle Physics: Concepts And Phenomena (Springer-Verlag, Berlin, Germany, 1990).

[57] I.I. Bigi and A.I. Sanda, CP Violation, 2nd edition (Cambridge University Press, Cambridge, UK, 2009).

[58] J. Bernabeu, G.C. Branco and M. Gronau, "CP restrictions on quark mass matrices," Phys. Lett. B 169, 243 (1986).

[59] H.K. Dreiner, H.E. Haber and S.P. Martin, "Two-component spinor techniques and Feynman rules for quantum field theory and supersymmetry," Phys. Rept. 494, 1 (2010) [arXiv:0812.1594 [hep-ph]].

[60] R.A. Horn and V.V. Sergeichuk, "Canonical forms for unitary congruence and *congruence," Linear and Multilinear Algebra 57, 777 (2009) arXiv:0710.1530 [math.RT]].

[61] W. Grimus, G. Ecker, "Basis transformations in generation space and a criterion for the existence of standard forms for unitarily congruent matrices," J. Phys. A 21, 2825 (1988).

[62] S.L. Glashow and S. Weinberg, "Natural conservation laws for neutral currents," Phys. Rev. D 15, 1958 (1977).

[63] E.A. Paschos, "Diagonal neutral currents," Phys. Rev. D 15, 1966 (1977).

[64] P.M. Ferreira and J.P. Silva, "Discrete and continuous symmetries in multi-Higgs-doublet models," Phys. Rev. D 78, 116007 (2008) arXiv:0809.2788 [hep-ph]].

[65] P.M. Ferreira, M. Maniatis, O. Nachtmann and J. P. Silva, "CP properties of symmetry-constrained two-Higgs-doublet models," JHEP 1008, 125 (2010) arXiv:1004.3207 [hep-ph]].

[66] W. Grimus and L. Lavoura, "Renormalization of the neutrino mass operators in the multi-Higgs-doublet standard model," Eur. Phys. J. C 39, (2005) 219 arXiv:hep-ph/0409231.

[67] P.M. Ferreira and D.R.T. Jones, "Bounds on scalar masses in two Higgs doublet models," JHEP 0908 (2009) 069 arXiv:0903.2856 [hep-ph]].

[68] W.-K. Tung, Group Theory in Physics (World Scientific, Singapore, 1985).

[69] K.N. Srinivasa Rao, The Rotation and Lorentz Groups and their Representations for Physicists (Wiley Eastern Limited, New Delhi, 1988).

[70] S.L. Altmann, Rotations, Quaternions, and Double Groups (Dover Publications, Inc., New York, NY, 2005). 